\title{
Extended Classical Over-Barrier Model for Collisions of Highly Charged Ions with Conducting and Insulating Surfaces
}

\author{
Jens J. Ducrée*, Fulvio Casali, and Uwe Thumm ${ }^{\dagger}$ \\ J. R. Macdonald Laboratory, Department of Physics, Kansas State University, Manhattan, \\ Kansas 66506-2604, USA
}

(March 5, 2018)

\begin{abstract}
We have extended the classical over-barrier model to simulate the neutralization dynamics of highly charged ions interacting under grazing incidence with conducting and insulating surfaces. Our calculations are based on simple model rates for resonant and Auger transitions. We include effects caused by the dielectric response of the target and, for insulators, localized surface charges. Characteristic deviations regarding the charge transfer processes from conducting and insulating targets to the ion are discussed. We find good agreement with previously published experimental data for the image energy gain of a variety of highly charged ions impinging on $\mathrm{Au}, \mathrm{Al}, \mathrm{LiF}$ and KI crystals.
\end{abstract}

PACS numbers: 34.50.Dy, 34.70.+e, 79.20.Rf

Typeset using REVTEX

*permanent address: Institut für Kernphysik, Wilhelm Klemm-Str. 9, D-48149 Münster

${ }^{\dagger}$ corresponding author 


\section{INTRODUCTION}

Within the past decade, a rapidly increasing number of research projects has been devoted to the investigation of interactions between highly charged ions (HCI) and surfaces (for recent reviews see [1 3]). These activities are of importance for present and future applications, such as semiconductor fabrication, nanostructure technology, and surface chemistry. They are also of interest to basic research due to the challenging interplay of fundamental electronic interactions to be considered in the detailed understanding of the highly complex interaction dynamics. By now, a certain level of consent has emerged with regard to charge exchange and ionization processes that take place before an HCI gets in close contact with a metal target surface, and a mainly classical approach, the "classical over-barrier model" (COM), first presented by Burgdörfer, Lerner, and Meyer «4,5], was found to adequately represent the most important physical aspects of the electron capture, recapture, and emission sequence [5 11].

Typically, an incident HCI captures several conduction band electrons at large distances from the surface into highly excited states which leads to the temporary formation of a "hollow ion" in front of the surface. At ion-surface distances that are smaller than or about equal to the classical radii of active HCI orbitals, the theoretical description becomes more difficult due to the strong perturbation of the initial electron distribution of the surface and the intricate molecular dynamics involved. For this reason, most first-principle calculations have been applied to incident ions in low charge states 12 15. For higher incident charge states the detailed quantum mechanical treatment is complicated by a large number of ionic states that are energetically degenerate with the target conduction band, and a first-principle approach remains a formidable task 16 19.

Most experiments with incident HCI have been performed for conducting and semiconducting surfaces with typical workfunctions of about $5 \mathrm{eV}$. These experiments focused on total electron yields [20], energy-resolved Auger [21 24] and X-ray 25,26] spectra, as well as deflection angles [27], and ion-neutralization [28 30] measurements. Recently, several 
experiments have been carried out, where HCI beams are incident on insulating surfaces, primarily on ionic crystals, such as LiF 31 34]. The unique band structure of LiF with a large workfunction of $12 \mathrm{eV}$ and a wide band gap of $14 \mathrm{eV}$ (Fig. 1) that elevates the antibinding $2 \mathrm{p}$-band above the vacuum level provides an interesting opportunity to scrutinize previous theories about the role of the conduction band in the charge exchange process. In contrast to metal surfaces, LiF and other insulating surfaces do not provide unoccupied conduction band states into which resonant loss from excited projectile states might occur, and pronounced differences are expected in the neutralization dynamics of HCIs in front of metal and insulating surfaces. Furthermore, the capture of electrons from an insulator leads to the local accumulation of positive surface charges that modify the charge transfer dynamics in comparison with metals. The capture-induced accumulation of localized charges on insulator surfaces has very recently been addressed in a few independent theoretical studies 35 37 and is a central aspect of the extentions to the COM discussed in this paper. Measurements on LiF surfaces [34] clearly exhibit the expected discrepancies with respect to conducting targets and can coherently be interpreted by a retarded onset for electron capture and characteristic deviations in the succeeding charge transfer sequence.

In this paper, we adopted the basic framework of the COM suggested by Burgdörfer et al. for the interaction of slow HCIs with metal surfaces [2, 4]. Different versions of COMs have been applied to successfully model charge exchange, energy gain, and trajectory effects in collisions of HCIs with atoms [38,39 and clusters $\left(\mathrm{C}_{60}\right)$ [6 8, 11]. Within the COM, the neutralization dynamics is described by means of an effective single electron potential which governs the classical motion of electrons that are going to be either resonantly captured into hydrogenic projectile levels or resonantly lost to unoccupied states of a metal target conduction band. For charge transfer to occur, an active electron must overcome the potential barrier between projectile nucleus and target surface. Electron transfer becomes classically possible if the electron initially occupies a state that lies above this potential barrier and if vacancies exist in the resonant final state into which the electron transits. As the projectile moves along a classical trajectory, both projectile and target levels experience variable level 
shifts and change their relative energetic positions with respect to the potential barrier, the position and height of which also changes as a function of the projectile-surface distance.

In order to perform simulations involving insulating surfaces we have extended the original COM [2, 4] by modifying the dielectric response of the surface to the external projectile charge and by including local surface charges. These local charges are built up on the insulator surface during the charge transfer sequence and decay on a time scale that is given by the conductivity of the insulator. The local surface charges influence active electrons and the projectile motion. In this work we will discuss the influence of these excess surface charges on electron transfer and projectile deflection in detail and comment on the recent and related theoretical work of Borisov et al. [35] and Hägg et al. [36].

We have organized this paper as follows. In Section III we review the main physical elements of our COM simulations, such as effective potentials (Sec. [IA), local workfunction changes (Sec. [1B), electronic transition rates (Sec. IIC), and the projectile motion (Sec. [ID). In Section [IIA we discuss in detail the neutralization dynamics in front of the surface in terms of the evolution of level occupations, potentials, projectile charge and motion above metal and insulator surfaces. In Section [11B we compare our results with previously published experimental and computed data on image energy gains of the projectile over a wide range of initial charge states. Our conclusions are contained in Section IV]. We use atomic units (a.u.) throughout this paper unless specified otherwise.

\section{OUTLINE OF THE EXTENDED COM}

\section{A. Potentials seen by an active electron}

Within the dynamical COM charge exchange is described in terms of classical charge currents between energetically shifted valence states of the target and shifted hydrogenic projectile levels. These continuous charge currents correspond to electronic transition rates for resonant capture and loss and occur as soon as the potential barrier $V_{b}$ of the total 
effective potential $V_{\text {tot }}$ drops below the target workfunction $W$. The total potential acting on an active electron is given by

$$
\begin{aligned}
V_{\text {tot }}\left(q_{p}, x, z, X_{p}, R, t\right) & =V_{\text {proj }}\left(q_{p}, x-X_{p}, z-R\right)+V_{i m, p}\left(q_{p}, x-X_{p}, z+R\right) \\
& +V_{\text {im,e }}(z)+V_{\text {local }}(x, z, t)
\end{aligned}
$$

where $x$ and $z$ will denote the electronic coordinates in the collision plane parallel and perpendicular to the surface-projected motion, respectively. The projectile distance from the surface is denoted by $R$. The projectile coordinate along the projection of the trajectory on the surface is $X_{p}$. The coordinate $x_{0}<0$ refers to the location on the surface where, at time $t_{0}$, charge transfer starts (Fig. 2). The origin of our coordinate system is located on the intersection of the topmost lattice plane (at $z=R=0$ ) and the collision plane. The jellium edge is located half a lattice constant above the uppermost lattice plane of the crystal. The potential saddle is located at $z_{b}$. The projectile is assumed to reach its point of closest approach to the surface at time $t=0$, and the surface projection of this point defines $x=X_{p}=0$. For our applications in this paper, it is sufficient to consider trajectories with surface projections along the [100] direction that intersect surface lattice points and define the projectile coordinate $Y_{p}=0$ (see Section IIIB2 below). A more general approach would average over many trajectories with $Y_{p}$ coordinates inside a surface unit cell.

The first term in (2.1) represents the interaction of the active electron with the projectile and is modeled by the Coulomb potential

$$
V_{p r o j}\left(q_{p}, x-X_{p}, z-R\right)=-\frac{q_{p}(R)}{\sqrt{\left(x-X_{p}\right)^{2}+(z-R)^{2}}} .
$$

The projectile charge

$$
q_{p}(R)=q_{n u c, p}-\sum_{n} a_{n}(R)
$$

depends on the nuclear charge $q_{n u c, p}$ of the HCI and the projectile shell occupations $a_{n}$. The index $n$ labels the principal quantum number of projectile shells.

Surface charge distributions produced in response to the external charges of the projectile and the active electron are included in (2.1) in form of the projectile image potential $V_{i m, p}$ and 
the self-image potential of the active electron $V_{i m, e}$. These potentials can be derived within linear response theory. Along an axis that is perpendicular to the surface and includes the projectile nucleus (i.e. for $x=X_{p}$ ), approximate expressions for these potentials, appropriate for grazing-incidence collisions, are given by [40]

$$
V_{i m, p}\left(q_{p}, 0, z+R\right)=\frac{2 q_{p}}{\pi v_{p}} \int_{0}^{\infty} d \omega \operatorname{Re}\left(\frac{1-\epsilon(\omega)}{1+\epsilon(\omega)}\right) \cdot K_{0}\left(\frac{\omega}{v_{p}}\left(z+R-2 z_{i m}\right)\right)
$$

and the self-image potential of the active electron

$$
V_{i m, e}(z)=-\frac{1}{\pi v_{p}} \int_{0}^{\infty} d \omega \operatorname{Re}\left(\frac{1-\epsilon(\omega)}{1+\epsilon(\omega)}\right) \cdot K_{0}\left(2 \frac{\omega}{v_{p}}\left(z-z_{i m}\right)\right)
$$

where $v_{p}$ denotes the projectile velocity and $K_{0}$ is a modified Bessel function.

Equations (2.4) and (2.5) refer to the dielectric response of the target material to a moving external charge in the undispersive approximation [40], for which the dielectric function $\epsilon(\vec{k}, \omega)$ is independent of the momentum $\vec{k}$. Following reference [41], we approximate the dielectric function

$$
\epsilon(\omega)=\epsilon_{\infty}+\frac{\epsilon_{0}-\epsilon_{\infty}}{1-\left(\omega / \omega_{0}\right)^{2}-i\left(\omega / \omega_{0}\right) \gamma}
$$

by its static $\left(\epsilon_{0}=\epsilon(0)\right)$ and optical $\left(\epsilon_{\infty}=\epsilon(\infty)\right)$ limits, the resonance frequency $\omega_{0}$, and a positive infinitesimal constant $\gamma$. Table I contains these constants for the two ionic crystals used in this work. Both image potentials are referred to the image plane at $z_{i m}>0$. In applications to metals, we identify the image plane with the jellium edge, such that $z_{i m}$ becomes equal to half a lattice constant. For ionic insulator crystal targets, we inserted the negative ion radius for $z_{i m}$, i.e. we assume that the induced positive image charge is located in the vicinity of the high density anionic electron cloud above the target. A similar independent study of the dynamic dielectric response of alkali halides was recently published by Hägg et al. [36] in which, as in (2.4), (2.5), and (2.6), the linear response theory result of Abajo and Echenique [40] was combined with a non-dispersive single-pole fit to the dielectric function. The main difference to our approach appears to be the use of a small but finite damping constant $\gamma$ in the work of Hägg et al.. We also note that Bárány and Setterlind 
[6] have developed a COM for capture from a dielectric sphere of radius $a$ and frequency independent, non-dispersive dielectric constant $\epsilon$. Their limit $a \rightarrow \infty$ corresponds to an insulating surface with simplified image interactions that include dielectric screening effects in terms of a frequency independent, multiplicative factor $(1-\epsilon) /(1+\epsilon)$.

For metal surfaces, we can take the limits $\epsilon \mapsto \infty$ in (2.4) and (2.5) and obtain the simple asymptotic forms 42

$$
\begin{aligned}
V_{i m, p}^{\text {metal }}\left(q_{p}, 0, z+R\right) & =\frac{q_{p}}{z+R-2 z_{i m}} \\
V_{i m, e}^{\text {metal }}(z) & =-\frac{1}{4\left(z-z_{i m}\right)} .
\end{aligned}
$$

In order to avoid the unphysical singularity of the electron self-image potential at $z=z_{i m}$, we truncate and steadily connect the total image potential to the bulk potential given by the lower limit of the metal conduction band by extending the constant bulk potential to a small distance outside $z_{i m}$. Our choice for $z_{i m}$ is a little larger than corresponding values obtained by fitting LDA calculations [43]. It is, however, sufficiently realistic within the overall precision of our model.

The capture of electrons from a solid leads to a linear surface charge distribution along the surface-projected path of the projectile (Fig. 2). For a metal surface, these local charge densities vanish instantaneously and do not influence the charge exchange sequence or the motion of the projectile due to high surface plasmon frequencies of the order of $10^{16} \mathrm{sec}^{-1}$. In contrast to metal surfaces, typical decay times for excess surface charges on insulators are by far too long to compensate local charge accumulations at the collision time scale. As a consequence the ion is followed by a linearly stretched trail of surface charges $q_{i}\left(x_{i}\right)$ generated at times $t_{i}, i=0, \ldots, i_{\max }\left(X_{p}\right)$ at locations $x_{i}$, for which the projectile is at distances $R\left(t_{i}\right)$ above the surface. The charge depends on the ion-surface distance $R$ through the implicit dependence of $x_{i}$ on $R$.

We assume the excess surface charges to decay exponentially with a time constant $\tau$. We can approximate $\tau$ by having charge currents $\vec{j}$ restore the electric neutrality as depicted in Figure 3. The driving force of these currents are electric fields $\vec{E}=\sigma \vec{j}$ caused by the local 
surface charges

$$
q_{i}\left(x_{i}, t\right)=q_{i}\left(x_{i}\right) \exp \left(-\frac{t-t_{i}}{\tau}\right), \quad t \geq t_{i}
$$

due to local charges $q_{i}\left(x_{i}\right)$ generated at times $t_{i}, i=0,1,2, \ldots$ when charge transfer took place. By choosing a hemisphere around the excess surface charge as Gaussian surface, Gauss' theorem leads to the decay time $\tau=(2 \pi \sigma)^{-1}$ in terms of the macroscopic conductivity $\sigma$. Typical values for $\sigma$ are $10^{7}(\Omega \mathrm{cm})^{-1}$ for metals and $\sigma \simeq 1 \cdot 10^{-6}(\Omega \mathrm{cm})^{-1}$ for ionic crystals, such as a LiF crystal, at room temperature. For LiF this simple estimate yields $\tau \simeq 10^{-7} \mathrm{~S}$ which lies about seven orders of magnitude above the collision time of typically $10^{-14} \mathrm{~s}$. This means that after capture sets in and while the HCI continues to interact with the surface, a positive linear charge distribution

$$
\begin{aligned}
\lambda(x, t) & =\sum_{i}^{i_{\max }\left(X_{p}\right)} q_{i}\left(x_{i}\right) \exp \left[-2 \pi \sigma\left(t-t_{i}\right)\right] \delta\left(x-x_{i}\right) \\
& \approx \sum_{i}^{i_{\max }\left(X_{p}\right)} q_{i}\left(x_{i}\right) \delta\left(x-x_{i}\right) \equiv \lambda(x)
\end{aligned}
$$

remains on the surface-projected projectile path on the ionic crystal's surface. This charge distribution pulls down the potential barrier and tends to repel the HCI from the surface. Its contribution to the total effective potential (2.1) amounts to

$$
V_{\text {local }}(x, z, t)=-\frac{\epsilon(0)-1}{\epsilon(0)+1}\left\{\frac{\left[q_{j, c e l l}\right]}{z}+\int_{x_{0}}^{X_{p}(t)} d x^{\prime} \frac{\lambda\left(x^{\prime}, t\right)}{\sqrt{z^{2}+\left(x^{\prime}-x\right)^{2}}}\right\} .
$$

The term $\left[q_{j, c e l l}\right]$ represents the integer charge withdrawn from the active surface cell by an active electron that crosses the barrier, e.g. $\left[q_{j, \text { cell }}\right]=1$ for $0<q_{j, \text { cell }} \leq 1$. The integral in (2.11) constitutes an average over previously transferred (non-integer) charges. For metal surfaces, both $\lambda$ and $V_{\text {local }}$ vanish.

The positive excess charge $\lambda\left(x^{\prime}\right) d x^{\prime}$ within a small interval $d x^{\prime}$ near $x^{\prime}$ polarizes the surrounding ionic crystal. This polarization effectively screens the local charge $\lambda\left(x^{\prime}\right) d x^{\prime}$ and is the origin of the Mott-Littleton correction [44,45] to the ground-state energy of the crystal. This screening correction is approximately included in (2.11) in terms of the 
static dielectric screening function $(\epsilon(0)-1) /(\epsilon(0)+1)$. A Mott-Littleton correction is also included in the work of Borisov et al. [35] and Hägg et al. [36]. Instead of our multiplicative screening factor in (2.11) Borisov et al. introduce this correction as an additive contribution to the attractive interaction of the active electron with the left-behind hole on the surface. The approach of Hägg et al. resembles our approximation in that the screening of the capture-induced surface charge is included as a multiplicative factor which asymptotically, for $z \rightarrow \infty$, becomes equal to a frequency independent dielectric screening function; it differs from (2.11) due to the inclusion of (i) dynamical screening of excess surface charges and (ii) fractional ionicity effects close to the surface in reference 36].

We note that in our version of the COM a continuous classical charge current is used to represent charge transfer. In our discussion of local surface charges, the discretized charge $q_{i}$ is used for convenience only and corresponds to the (small) portion of an elementary charge that is transferred during one timestep of the numerical propagation (see Section ПC4, below). In contrast, Borisov et al. [35] and Hägg et al. [36] enforce charge quantization and consider local excess surface charges of at least one positive elementary charge.

\section{B. Local workfunction}

We now examine the dynamic change of the local workfunction while the projectile draws a certain amount of charge from a specific surface atom on an insulating surface. In order to estimate the local workfunction of an ionic crystal, we assume that target electrons are captured from an anion on the surface lattice. The energy necessary to remove a loosely

bound valence electron from a surface anion can be approximated by the affinity $E_{\text {bind }}^{q}$ of the free anion (3.4 eV for free $\mathrm{F}^{-}$ions) and by adding the interactions of the detaching electron with all other target ions as a Madelung-background potential $V_{\text {Mad,bg }}$ (Fig. 凹). This leads to the workfunction

$$
W\left(r_{\text {anion }}^{q}\right)=E_{\text {bind }}^{q}+V_{\text {Mad,bg }}\left(r_{\text {anion }}^{q}\right) .
$$


We evaluated $V_{\text {Mad,bg }}$ at the mean radius $r_{\text {anion }}^{q}$ of the outermost ionic $n \ell$-shell $(n=2, \ell=1$ for $\mathrm{LiF}$ ), directly above the anion's lattice site,

$$
V_{\text {Mad,bg }}\left(r_{\text {anion }}^{q}\right)=\sum_{j} \frac{Q_{j}}{\left|\overrightarrow{R_{j}}-r_{\text {anion }}^{q} \hat{e}_{z}\right|}
$$

where $\hat{e}_{z}$ is a unit vector along the positive $\mathrm{z}$ axis. Since the contribution of the active surface anion is included via its binding energy, the active anion is exempted in the Madelung sum over all lattice sites $\vec{R}_{j}$ in (2.13). Without taking screening effects into account, we assume $Q_{j}=-1$ for all anionic charges and $Q_{j}=1$ for all cathionic charges in (2.13). Equation (2.12) yields values of $11.65 \mathrm{eV}$ for $\mathrm{LiF}$ and $8.21 \mathrm{eV}$ for $\mathrm{KI}$ and thus reproduces the experimentally determined workfunctions (12 eV for $\mathrm{LiF}$ and $8.2 \mathrm{eV}$ for KI [46]) sufficiently well within the overall accuracy of our approach.

In order to include the effect of the net capture-induced local charge $q_{j, \text { cell }}>0$ residing within the active surface lattice cell $j$ on the local workfunction, we added $q_{j, \text { cell }}$ to the original anionic charge $Q_{j}=-1$ and obtain the new charge $q=Q_{j}+q_{j, \text { cell }}$, a corresponding new ionic binding energy $E_{\text {bind }}^{q}$ and, by using the Cowan code [47], an adjusted ionic radius $r_{\text {anion }}^{q}$. Inserting these quantities into both terms of (2.12) supplies the adjusted workfunction $W\left(r_{\text {anion }}^{q}\right)$. Since the dynamical COM simulates continuous charge currents, we interpolate between discrete $r_{\text {anion }}^{q}$-values. In this way we can compute the local workfunction (2.12) as a function of the capture-induced local surface charge $q_{j, \text { cell }}$ within the active anion's unit cell. In contrast to the approach of Hägg et al. [36], we do not include fractional ionicity and screening effects in the local workfunction. In agreement with the papers of Borisov et al. [35] and Hägg et al. [36], our local workfunction includes a Madelung sum for the interaction of the detaching/ionizing negative charge with the ionic crystal and an additional term for the interaction with the excess surface charge on the active anion site. However, in contrast to these authors, we relate the additional term to the affinity of a free anion of charge $Q_{j}=-1$ and interpolate (using atomic ionization potentials) to effective charges $q>-1$, as dictated by the non-charge-quantized version of the COM. For LiF, we allow for at most one electron to be captured from an active $\mathrm{F}^{-}$site, such that $q_{j, \text { cell }} \leq 1$. For KI, we take the 
large number of outer shell electrons on $\mathrm{I}^{-}$in account by removing this restriction.

In Figure 5 we show the various contributions to the total electronic potential (2.1) for $50 \mathrm{keV} \mathrm{Xe^{15+ }}$ ions that approach a LiF surface at a grazing angle of $1^{\circ}$. The potentials are displayed along an axis perpendicular to the surface that includes the projectile nucleus. The projectile is on the incident part of the trajectory at a distance $R=10 \mathrm{a} . \mathrm{u}$. in front of the surface. The remaining projectile charge $q_{p}(R=10)$ amounts to 2.8, i.e. $\int_{x_{0}}^{X_{p}} \lambda(x) d x=12.2$.

\section{Transition rates}

In this section, we summarize the approximations that lead to simple analytical expressions for resonant and Auger transition rates, closely following references [2, [1]. These rates are then combined in form of a system of coupled classical rate equations in order to describe the occupation dynamics of projectile levels.

\section{Resonant gain}

We represent the electronic structure of the projectile by its spectrum of energy levels $\varepsilon_{n}(R)$ and their occupations $a_{n}(R)$. Both quantities change during the motion of the projectile. We assume hydrogenic shells with binding energies

$$
\varepsilon_{n}(R)=-\frac{1}{2}\left(\frac{q_{e f f, n}(R)}{n}\right)^{2}
$$

that depend on the effective charges

$$
q_{e f f, n}(R)=q_{n u c, p}-\sum_{n^{\prime}} S_{n, n^{\prime}} a_{n^{\prime}}(R)
$$

The matrix $S_{n, n^{\prime}}$ accounts for screening effects and is determined under the simplifying assumption of full inner screening and no screening by outer and equivalent electrons $\left(S_{n, n^{\prime}}=\right.$ 1 for $n>n^{\prime}$ and $S_{n, n^{\prime}}=0$ otherwise). The classical model of a continuous charge flow over the potential barrier in conjunction with discrete energy levels (2.14) requires the definition 
of energy bins. We designate energy bins by $\left[\varepsilon_{n}(R)\right]$. Each bin is attributed to a projectile shell $n$ and includes electronic energies $\varepsilon$ within the interval

$$
\left[\varepsilon_{n}(R)\right] \equiv\left[\frac{1}{2}\left(\varepsilon_{n}-\varepsilon_{n-1}\right)+V_{i m, p}+V_{i m, e}+V_{l o c a l}, \frac{1}{2}\left(\varepsilon_{n+1}-\varepsilon_{n}\right)+V_{i m, p}+V_{i m, e}+V_{l o c a l}\right]
$$

including corrections for level shifts due to image charge interactions and localized surface charges. Thus the charge current $I$ transferred classically from the surface into the energy bin $\left[\varepsilon_{n}(R)\right]$ is considered to feed the $n$ 'th energetically shifted shell of the projectile.

The resonant gain current (i.e. the resonant gain rate) $I_{n}^{r g}$ from the surface into a particular $n$-manifold of the HCI is given by the product

$$
I_{n}^{r g}(R, t)=\sigma(R) j_{n}(R, t)
$$

of the current density

$$
j_{n}(z)=\frac{1}{4} \int_{\max \left(V_{b}, \varepsilon_{n-1 / 2}\right)}^{\min \left(-W, \varepsilon_{n+1 / 2}\right)} d E D(E) \sqrt{2\left(E-V_{b}\right)},
$$

and the cross section $\sigma$. The conduction band density of states in free-electron-gas approximation is given by $D(E)=V \sqrt{2} / \pi^{2} \sqrt{E-V_{0}}$, where $-V_{0}$ is the lower valence band limit with respect to the ionization threshold (cf. Fig. 1). Values for the targets investigated in this work are $V_{0}=10.6,10.9,15.9,14$ and $10.5 \mathrm{eV}$, respectively for $\mathrm{Au}$ (polycrystalline) [10,48], $\mathrm{Au}(110)$ [10,48], $\mathrm{Al}$ [48], LiF [46], and $\mathrm{KI}$ [49]. $V$ is a volume which we assume to be one $(\text { a.u. })^{3}$ for the following. The factor $\sqrt{2\left(E-V_{b}\right)}$ is the classical velocity of active electrons while passing the potential barrier. The energetic bottom of the conduction band lies $V_{0}$ below the ionization threshold, and the geometrical factor $1 / 4$ in (2.18) relates the isotropic density of states, $D(E)$, to the electron current along the positive surface normal. The cross section

$$
\sigma=\pi\left(\frac{\Delta x}{2}\right)^{2}
$$

is equal to the classically allowed area over the potential saddle through which the current representing active electrons needs to flow. The effective width $\Delta x(t)=\left|x_{1}(t)-x_{2}(t)\right|$ of the saddle at any time is given implicitly by the two solutions $x_{1}$ and $x_{2}$ of 


$$
\varepsilon_{n}(R)+V_{i m, p}\left(q_{p}, x-X_{p}, z_{b}+R\right)+V_{i m, e}\left(z_{b}\right)+V_{\text {local }}\left(x, z_{b}, t\right)=V_{\text {tot }}\left(q_{p}, x, z_{b}, X_{p}, R, t\right)
$$

where, as in (2.4), the nuclear image potential is evaluated on the axis $x=X_{p}$.

We note that the valence band density of states of an ionic crystal per se is poorly represented with the free electron gas model. Hägg et al. [36] used classical Monte Carlo techniques in order to simulate the over-barrier dynamics of target electrons that are released from an anionic center by the highly charged projectile. Their Monte Carlo study indicates that the electron is effectively captured from LiF at a projectile surface distance that is about 3 a.u. closer to the surface than the onset of classical electron capture across the potential barrier. Interestingly, our numerical results, including capture rates modeled with reference to the free electron density of states, also indicate a delayed onset between the initiation of a classical over-barrier current and the projectile-surface distance where one elementary charge has been transferred (see Section IIIA below). Furthermore, we point out that previous dynamical COM studies on electron capture in collisions with $\mathrm{C}_{60}$ [7] have shown a rather weak dependence of final projectile charge states and critical capture radii on variations in the resonant capture rates. We therefore conclude, that within the overall accuracy of the COM and in view of the narrow valence band of ionic crystals, the free electron gas model is sufficiently realistic for providing acceptable estimates for resonant capture rates. The agreement of our simulated projectile energy gains with experiments for ionic crystal surfaces provides further support for this approximation (see Section IIIB2, below).

\section{Resonant loss}

The rate of electron loss from atomic energy levels into unoccupied bulk levels can be obtained from the electron's orbital frequency of revolution

$$
f_{n}=\frac{q_{e f f, n}^{2}}{2 \pi n^{3}}
$$

multiplied with its probability to hit the saddle region that is approximately given by 


$$
P\left(\varepsilon_{n}\right)=\left[z_{\text {crit }}\left(\varepsilon_{n}\right)-z\right] / z_{\text {crit }}\left(\varepsilon_{n}\right)
$$

where $z_{\text {crit }}\left(\varepsilon_{n}\right)$ denotes the critical distance where the first electron capture occurs into shell $n$ 壮,

$$
I_{n}^{r l}(z)=f_{n} \cdot P\left(\varepsilon_{n}\right)
$$

For insulators, particularly for LiF with no unoccupied band levels below the vacuum level, resonant loss is irrelevant, and $I_{n}^{r l}=0$.

\section{Auger processes}

Intraatomic Auger transitions induce small changes in the projectile occupation during the interaction with the surface. Following reference [4] we express the Auger rates by a simple analytic fit through data points

$$
\Gamma_{n_{i}, n_{f}}=\frac{5.06 \cdot 10^{-3}}{\left(n_{i}-n_{f}\right)^{3.46}}
$$

that have been calculated with the Cowan code [47 for fast transitions between two given shells $n_{i}$ and $n_{f}$.

\section{Rate equations}

The dynamically varying projectile populations are obtained as solutions to the coupled set of classical rate equations

$$
\frac{d a_{n}}{d t}=\Theta\left(A_{n}-a_{n}\right) \Gamma_{n}^{r g}-a_{n} \Gamma_{n}^{r l}+w_{f, n} \sum_{n^{\prime}>n} \Gamma_{n^{\prime}, n} w_{i, n^{\prime}}-2 w_{i, n} \sum_{n^{\prime}<n} \Gamma_{n, n^{\prime}} w_{f, n^{\prime}}
$$

where the degeneracy of shell $n$ is given by $A_{n}=2 n^{2}$. $\Theta$ is the unit step function. The (empirical) statistical factor $w_{f, n}=1 /\left(1+1.5 a_{n}\right)$ corrects for the decrease in Auger transition rates due to increasing populations $a_{n}$ of the final level. The statistical factor $w_{i, n}=\frac{1}{2} a_{n}\left(a_{n}-\right.$ 1) takes the equivalence of electrons in the initial shell into account. 


\section{Projectile motion}

Before reaching the first critical over-barrier radius, the motion of the incoming HCI is solely affected by its attractive self-image force. For metal surfaces this force is given by

$$
F_{i m, p-p}(R)=-\left(\frac{q_{p}}{2\left(R-z_{i m}\right)}\right)^{2}
$$

and for insulating surfaces by the derivative of (2.4). After the projectile has reached the first critical over-barrier distance, charge transfer begins and, for insulators, the self-image force (2.26) starts to compete with the repulsive force created by localized surface charges (2.11). For grazing collisions, the latter force is weak, due to the large projectile velocity component parallel to the surface, which rapidly increases the distance between previously created surface charges and the HCI.

At distances $R$ smaller than the largest radius of occupied atomic orbitals, $\langle r\rangle_{n}$, the electron clouds of the incoming HCI and the surface ions begin to penetrate each other. The accurate description of this situation would require detailed quantum dynamical calculations, which are far beyond the overall simplistic nature and accuracy of the COM. In order to determine the classical motion of the projectile, we employ the Thomas-Fermi model and use the Thomas-Fermi-Molière potential energy [50],

$$
W_{T F M}(r)=\frac{q_{n u c, p} \cdot q_{n u c, t}}{r} \cdot \phi\left(\frac{r}{a}\right)
$$

This interatomic potential includes the Coulomb repulsion between the two nuclear charges $q_{n u c, p}$ and $q_{n u c, t}$ of the HCI and a target atom, respectively, and a screening function $\phi$ that depends on the internuclear distance $r$ scaled by the screening length $a$,

$$
\begin{aligned}
\phi\left(\frac{r}{a}\right) & =\sum_{i=1}^{3} \alpha_{i} \exp \left(-\beta_{i} \frac{r}{a}\right) \\
a & =0.88534 / \sqrt{q_{n u c, p}^{2 / 3}+q_{n u c, t}^{2 / 3}},
\end{aligned}
$$

with $\left\{\alpha_{i}\right\}=\{0.35,0.55,0.10\},\left\{\beta_{i}\right\}=\{0.3,1.2,6.0\}$. 
For for small perpendicular projectile velocity components, as typically given in grazing incidence collisions, the interaction of the HCI with an array of surface atoms or ions is well represented by taking the planar average of (2.27) [51,

$$
\begin{array}{r}
W_{T F M}(R)=\frac{2 \pi a q_{n u c, p} q_{n u c, t}}{d^{2}} \phi^{\prime}\left(\frac{R}{a}\right) \\
\phi^{\prime}\left(\frac{R}{a}\right)=\sum_{i=1}^{3} \frac{\alpha_{i}}{\beta_{i}} \exp \left(-\beta_{i} \frac{R}{a}\right)
\end{array}
$$

where, for simplicity, we have assumed a square lattice with lattice constant $d$.

For ionic crystals that are composed of two different ion species, we apply (2.29) separately to surface lattices of anions and cathions. This results in the planar averaged potential

$$
W_{T F M}(R)=W_{T F M}^{\text {anion }}(R)+W_{T F M}^{\text {cathion }}(R)
$$

where $W_{T F M}^{\text {anion }}+W_{T F M}^{\text {cathion }}$ are constructed according to (2.29) with $q_{n u c, t}$ replaced by the respective nuclear charges of anions and cathions and with the distance $d$ between anions or between cathions, respectively.

The force exerted on the projectile by the capture-induced surface charge distribution (2.10) is repulsive with a parallel component that accelerates the projectile in positive $\mathrm{x}^{-}$ direction. It is given by

$$
\vec{F}_{\text {local }}\left(X_{p}, R\right)=\frac{\epsilon(0)-1}{\epsilon(0)+1} q_{p} \int_{x_{0}}^{X_{p}} d x^{\prime} \frac{\lambda\left(x^{\prime}, t\right)\left(R, X_{p}-x^{\prime}\right)}{\left(R^{2}+\left(X_{p}-x^{\prime}\right)^{2}\right)^{3 / 2}} .
$$

The factor $(\epsilon(0)-1) /(\epsilon(0)+1)$ accounts for the static dielectric screening (cf. equ. (2.11)). The effect of this force on the projectile motion parallel to the surface is small. For $30 \mathrm{keV}$ $\mathrm{Xe}^{15+}$ incident under a grazing angle of $1^{\circ}$ on $\mathrm{LiF}$, it changes the parallel component of the projectile velocity by $0.2 \%$.

The net force on the projectile is now given by the negative gradient of $W_{T F M}$, the projectile self-image force (2.26), and (2.31),

$$
\vec{F}_{\text {net }}\left(X_{p}, R\right)=\left\{-\frac{d}{d R}\left(W_{T F M}(R)\right)+F_{\text {im }, p-p}(R)\right\} \hat{e}_{z}+\vec{F}_{\text {local }}\left(X_{p}, R\right) .
$$




\section{NUMERICAL RESULTS AND DISCUSSION}

In this section we are going to discuss the pronounced differences in the above-surface neutralization dynamics between insulating and conducting targets as predicted by our simulations. All electronic interactions depend strongly on the projectile's position on its trajectory $\left(X_{p}(t), R(t)\right)$, which, in turn, depends on the charge state evolution $q_{p}(t)$ and, for insulator targets, on the trail of positive excess surface charges. Due to this coupling of nuclear and electronic degrees of freedom, the reconstruction of measured projectile deflection angles and projectile energy gains will supply support for the specific interaction model implemented in our simulation. Even though it is not yet possible to extract direct evidence from the experimental data for the time evolution of many quantities included in our simulation, such as shell populations and level shifts (Sec. IIIA), good agreement between energy gain measurements and theory (Sec. IIIB) supports the validity of our model assumptions.

\section{A. Interaction dynamics}

Fig. 6a shows the simulated projectile charge evolution for $\mathrm{Xe}^{15+}$ ions colliding with an Al target in one case, and a LiF crystal in the other. The incident projectile energy is $50 \mathrm{keV}$ at a grazing incidence angle of $1^{\circ}$. For $\mathrm{Al}$, the first critical distance for classical over-barrier capture is $R_{c} \approx 38$. In the case of $\mathrm{LiF}$, the interplay of the large workfunction and image charges, which compared with the Al target are reduced by the altered dielectric response function, effectively shifts the onset of charge transfer by about 10 a.u. to $R_{c} \approx 30$. Our version of the COM does not impose charge quantization. By rounding to nearest integer charges, the neutralization sequence is therefore completed when the projectile charge becomes smaller than 0.5 . In comparison with $\mathrm{LiF}$, we find that for the same ion-surface distance $R$ the early onset of electron transfer on $\mathrm{Al}$ leads to smaller projectile charges above the metal target.

Differences in the time evolution of resonant gain processes become apparent by com- 
paring resonant gain rates (Fig. 6b and Fig. 7a) or projectile level occupations (Fig. 6lc and Fig. 7 $\mathrm{b}$ ) for the two targets and incident $\mathrm{Xe}^{15+}$ ions. On the $\mathrm{Al}$ target, the $\mathrm{n}=18$ shell of the projectile becomes strongly populated with filling rates of the order of $10^{14} \mathrm{~s}^{-1}$ (Fig. 7). For $\mathrm{LiF}$, resonant gain transfer occurs into the $\mathrm{n}=14,13$, and 12 shells. The sharp maxima of the gain rates on $\mathrm{LiF}$ exceed the metal rates by almost two orders of magnitude. The average neutralization rates, however, i.e. the slopes of the corresponding shell occupations (Fig. 6a and (Fig. 7b), are only slightly higher in LiF. The interruption of the projectile neutralization between $R=19$ and $R=21$ coincides with the transient increase of $V_{b}$ above $W$ in Fig. $8(\mathrm{~b})$. Resonant loss processes are either forbidden ( $\mathrm{LiF}$ ) or contribute with negligible rates $(\mathrm{Al})$. For the $\mathrm{Al}$ target, shells below the resonantly populated level $\mathrm{n}=18$ are populated in Auger transitions.

The regularly spaced spikes in the resonant gain rate for $\mathrm{Xe}^{15+}$ impinging on LiF (Fig. 6b) originate in the capture-induced local surface charges. As the surface-projected path of the ion enters a new surface cell containing a single fluorine $\mathrm{F}^{-}$ion, the workfunction is reset to its original value $W=12 \mathrm{eV}$, such that the local Fermi level suddenly moves upwards thereby stimulating over-barrier capture. The corresponding workfunction changes amount to up to $6 \mathrm{eV}$ (Fig. $8 \mathrm{~b}$ ), whereas oscillations in the barrier height $V_{b}$ of the potential saddle remain comparatively small with an amplitude of less than $1 \mathrm{eV}$ as the ion travels over the surface cell boundaries. This relatively inert behavior of $V_{b}$ can be explained by the moderate influence of the local surface charges on the total potential $V_{\text {tot }}$ near the saddle position $z_{b}$ which is situated typically a few a.u. in front of the first bulk layer (see also Fig. 5). We note that, if capture from a given anion proceeded, the local workfunction would increase by about $10 \mathrm{eV}$ per unit capture-induced surface charge. However, due to the high transfer rates of up to $10^{16} \mathrm{~s}^{-1}$ the continuous current of negative charge is quickly cut off at the moment when the Fermi level is shifted below the saddle point $V_{b}$ ("over-barrier cut-off"). In other words, the local workfunction change (i.e. the shift of the Fermi level towards lower energies and below $V_{b}$ ) generated by capture-induced surface charges produces the peaked structures in the resonant gain rates. For the Al target, the absence of local surface charges 
results in a comparatively steady evolution of the gap between $V_{b}$ and $W$ (Fig. Ba) and results in the mostly smooth development of the dominant resonant gain rates in Figure $7 \mathrm{a}$.

Considering the characteristic discrepancies in the resonant exchange mechanisms, it is surprising that the average rate of neutralization is very similar for both targets. The effects of the low alkali halide workfunction on the onset of charge exchange, the reduced dielectric response of the insulator, and local surface charges appear to be counterproductive. As will be shown below (Sec. [IIB), this interplay is also related to the strikingly small differences between the image energy gains of a particular HCI on LiF and Al targets.

In Figure 9 we compare ion trajectories for grazingly incident $20 \mathrm{keV} \mathrm{Xe}^{15+}$ on $\mathrm{Al}$ and LiF surfaces. At large distances, the magnitude of the perpendicular velocity component $\left|v_{z}\right|$ steadily increases due to the attractive projectile self-image force. The short range TFMpotential in (2.27) causes the inversion of the trajectory in a small region that measures about 2 a.u. relative to the vertex of the trajectory leading to nearly specular reflection. For the LiF target the attenuated dielectric response of the insulator weakens the image attraction in comparison with a metal target. We simulated this effect in a separate calculation for $\mathrm{LiF}$ where we replaced the insulator specific dielectric response in the projectile (self-) image interactions by the asymptotic response of a perfect metal, taking (2.26) and the limit $\epsilon \rightarrow \infty$ in (2.4). This yields a noticeable change in the ion trajectory. Before capture sets in at large $R$, only the image force acts on the HCI and the "metal dielectric response" trajectory for LiF nearly coincides with the ion trajectory in front of Al. The replacement of the insulator-specific dielectric response by the metallic response on LiF moves the onset of charge transfer 13 a.u. closer to the surface. This shifts the potential barrier upwards due to the more repulsive (unscreened) projectile image term (2.4), thus reduces the amount of charge captured, and more than doubles the overall energy gain above the target, clearly leading away from both our dynamical COM with insulator-specific response and experimental results (see Sec. [IIB).

In another separate simulation we have eliminated all effects due to the capture-induced positive surface charge distribution on LiF (Fig. 9). The local surface charges add to the 
projectile repulsion near the surface. The vertex is now located about 0.1 a.u. closer to the

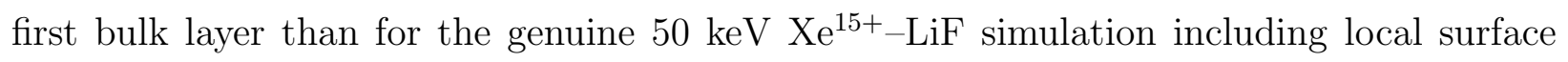
charges. This small shift suggests that the direct influence of these surface charges on the projectile trajectory is rather small. The large discrepancy of $12.8 \mathrm{eV}$ in the image energy gains between both simulations (see Section [11B2) originates mainly from deviations in the neutralization dynamics leading to a higher average projectile charge in front of the surface when local surface charges are disabled. This can be understood by considering that the component $V_{\text {local }}$ in (2.11) pulls down the potential barrier (2.1) and also the projectile energy levels (2.16) and thus counteracts electron loss due to level promotion into the continuum (if an occupied level gets promoted to the continuum, we assume that the level is instantaneously ionized).

The distance of closest approach to the surface under grazing incidence is determined by the initial velocity component perpendicular to the surface, the total kinetic energy gain of the HCI at the turning point, and by the composition of the target material via the screened interatomic interaction (2.27). Our simulations yield turning points at $R=1.1$ and 1.3 for $\mathrm{Xe}^{15+}$ ions impinging on $\mathrm{Al}$ and $\mathrm{LiF}$, respectively, at an incident energy of $30 \mathrm{keV}$ and an incidence grazing angle of $1^{\circ}$.

\section{B. Image energy gains}

After having presented detailed results on the interaction dynamics in the previous section, we are now going to demonstrate that the extended dynamical COM can quite accurately reproduce previously published (measured and simulated) data on image energy gains for both conducting and insulating crystals over a wide range of initial projectile charge states. Our simulations as well as recent experiments [29] show that the neutralization of the HCI is completed prior its reflection for a wide range of initial projectile charge states. The inversion of the perpendicular velocity component $v_{\perp}$ takes place at a distance

of a few atomic units above the surface (Fig. 9), and the measurable difference between the 
asymptotic incident and reflection angles of an ion beam can be straightforwardly correlated to the net image energy gain [52,27].

\section{Metals}

Image energy gains of a HCI impinging on metal surfaces are characterized by an approximate $q_{p}^{3 / 2}$-increase with the initial projectile charge state $q_{p}$ [9, 10,27]. A lower limit for the energy gain can be deduced by assuming that the projectile is instantaneously and completely neutralized at the first critical distance $R_{c} \simeq \sqrt{8 q_{p}+2} /(2 W)$ [53]. We shall refer to this estimate as "simple COM". The energy gain for large $q_{p}$ is then given by the analytical formula

$$
\Delta E=\frac{q_{p}^{2}}{4 R_{c}}=\frac{W q_{p}^{3 / 2}}{4 \sqrt{2}} .
$$

Consequently, $\Delta E / W$ should be independent of the target material.

The simple COM can be improved by letting one electron transfer to the HCI each time the over-barrier condition is fulfilled at consecutive critical radii for the first, second, etc. capture. This version of the COM is called the "staircase model" [10]. In contrast to the dynamical COM (Sec. ПA $)$, in the simple and staircase model the charge transfer current is quantized. For energy gains of $\mathrm{Xe}^{q}+$ projectiles on an $\mathrm{Al}$ surface, the staircase model almost coincides for all initial charges $q_{p}$ with the dynamical COM, and the simple model predicts, as expected, lower energy gains for all $q_{p}$ (Fig. 10). Except for the highest charge states, both, the staircase and dynamical COMs agree with the experimental gains of Winter et al. [52], even though the more elaborate dynamical COM employs transition rates that depend on the width and depth of the potential saddle. The simple model underestimates the measured energy gain, except for the highest charge states, where agreement with the experiment may be fortuitous. Except for the simple COM, all simulations intersect the experimental error bars for charge states $q_{p} \leq 30$. All simulations show the general $q_{p}^{3 / 2}$-trend.

The deviation in the experimental data from the approximate $q_{p}^{3 / 2}$-proportionality of the energy gain in all COM versions above $q_{p} \simeq 26$ (Fig. 10) has been scrutinized by Lemell et al. 
[10]. The authors rule out both saturation effects in the surface charge density fluctuations induced by the HCI at $R=R_{c}$ and effects due to the parallel velocity of the HCI. They

conclude that the measured deviation from the $q_{p}^{3 / 2}$-proportionality is due to incomplete screening of outer shells at decreasing $R$ which, for high initial projectile charges, leads to a faster decrease of the effective projectile charge and, therefore, to a diminished increase in the energy gain as a function of $q_{p}$. However, as far as we know, the initial charge state $q_{p}$ at which the experimentally observed plateau appears has not yet been reliably calculated within any COM (see also the review article of Winter [29] and references therein). Figure 10 also shows that the staircase COM calculation of Lemell et al. [10] agrees with our results.

Kurz et al. [54 have analyzed total electron yields for higher charge states as a function of the inverse projectile velocity. Their data for $\mathrm{Xe}^{q+}, q=34 \ldots 50$ and $\mathrm{Th}^{q+}, q=61 \ldots 79$ on gold surfaces under perpendicular incidence provide, if at all, weak evidence for a deviation of the energy gain from the $q^{3 / 2}$-proportionality (Fig. 11). We note that the experimental method of reference [54] is prone to larger errors than the deflection angle method [52]. Our dynamical COM data for Thorium are near the upper end of the experimental error bars.

In Figure 12 we compare our dynamical COM results for $150 \mathrm{keV}$ ions on Au with energy gains measured by Meyer et al. [28] and with the COM simulation of Lemell et al. [10]. The experimental results show good overall agreement with our calculations for both projectiles but fall systematically short of the dynamical COM values above $q_{p} \simeq 30$.

\section{Insulators}

Energy gains for $50 \mathrm{keV}$ Xe ions directed under a grazing incidence angle of $1^{\circ}$ on alkali halide crystals (LiF and KI) have been measured recently by Auth et al. 31,33 by using the deflection angle method [52]. Our extended dynamical COM simulations agree with experiment for the KI target for $q_{p}<17$ (Fig. 13). However, experiment and simulation tend to deviate in a systematic way for the LiF target, where, for low and intermediate incident charge states, the measured values slightly exceed our simulations. We tried to 
identify an adjustable parameter in order to further improve the agreement with experiment for both targets. In the oncoming paragraphs we will discuss several of the effects that appear in our insulator extension of the COM and use $\mathrm{Xe}^{15+}$ on LiF and KI, with an energy gains of $43.6 \mathrm{eV}$ and $31.8 \mathrm{eV}$, as a reference.

At first we take a closer look at effects that are induced by the surface charges. We observe that the restriction of one removable charge per $\mathrm{F}^{-}$ion (which we did not apply to iodine for its vast number of outer shell electrons) lowers the energy gain by $1.6 \mathrm{eV}$. Furthermore, disregarding all capture-induced surface charges (cf. curves labeled $q_{\text {local }}=0$ in Fig. 13) increases the energy gain of $\mathrm{Xe}^{15+}$ on $\mathrm{LiF}$ to $56.3 \mathrm{eV}$. For the KI target, however, the neglect of surface charges increases the energy gain to $37.0 \mathrm{eV}$ for incident $\mathrm{Xe}{ }^{15+}$ which lies above the experimental error bars. Our numerical results show the expected increase for energy gains at all charge states if we discard surface charges. For the KI target, the inclusion of capture-induced surface charges improves the agreement between simulated and measured energy gains.

With respect to the ionic conductivities, we note that $\sigma$ has to be increased by more than six orders of magnitude in order to induce any significant change in the energy shifts. Despite our crude estimates for $\sigma$ and the order-of-magnitude derivation of the time constant $\tau$ in (2.9), we can therefore exclude life-time effects of capture-induced surface charges on the simulated energy gains.

The image plane is located at one anionic radius above the uppermost bulk layer. This choice constitutes an upper limit for $z_{i m}$. Alternatively, as an accurate value is difficult to assess and the concept of an image plane is not well defined for ionic crystals, one could use $z_{i m}$ as an adjustable parameter. Placing the charge distribution at the topmost lattice plane diminishes the image energy for $\mathrm{Xe}^{15+}$ by $1.6 \mathrm{eV}$ on LiF. For KI the energy gain slightly increases by $0.6 \mathrm{eV}$.

We have performed all simulations with free-electron densities of states and a constant volume factor $V=1$ in the transition rates (2.17), which is a rather poor approximation for an ionic crystal and may not give sufficient credit to the characteristics of a particular crystal; 
the negative fluorine ion possesses six $2 p$-electrons, whereas iodine ion holds a large number of loosely bound electrons. In an attempt to work this information into the simulation, we reduced the resonant gain rates (2.17) for the LiF target by a factor of 5 . As a result we find that these modified rates lead to energy gains on LiF that lie inside the experimental error bars for all charge states. In a more realistic representation of the target electronic structure, more attention must be given to the valence electrons of the anions. The above-mentioned discrepancies for KI and higher charges of the incident projectile may be related to the simplified representation of the target electronic structure inherent in our implementation of the dynamic COM.

As explained in Section IIA, our simulations were limited to trajectories with $Y_{p}=0$, for which the collision plane intersects anionic and cathionic nuclei along the [100] direction. Since in surface scattering experiments the incident ion beam illuminates a surface area that is large compared with a surface unit cell, we addressed the sensitivity of our simulated energy gains to changes in $Y_{p}$. For $Y_{p}=0.5 d$, corresponding to surface-projected trajectories half way between ionic rows, we find for $30 \mathrm{keV} \mathrm{Xe}{ }^{15+}$ projectiles incident under $1^{\circ}$ on $\mathrm{LiF}$ a kinetic energy gain of $44.6 \mathrm{eV}$, compared to $43.6 \mathrm{eV}$ for trajectories with $Y_{p}=0$ and an experimental value [33] of $53.2 \mathrm{eV}$. The slightly larger energy gain is consistent with the increased average distance of capture-induced surface charges from the projectile. The change in energy gain with $Y_{p}$ is sufficiently small such that, within the overall accuracy of our calculation, we do not need to include a time-consuming average over trajectories with different $Y_{p}$ in our simulation.

\section{CONCLUSIONS}

In this work we applied and discussed extensions to the classical over-barrier model that include insulator specific effects such as capture-induced local surface charges, local workfunction changes, and the dielectric response of the target. A detailed study of the interaction mechanism has been presented in terms of the time evolution of projectile level 
occupations, transition rates, and several other quantities involved in the neutralization process.

Our results are in good agreement with previously published experimental data for highly charged ions impinging on two different alkali halide ionic crystals. In order to verify the basic framework of our implementation of the dynamical COM, we have disabled all effects related to insulators and found good agreement with energy gain measurements for a variety of incident ion charge states and metal targets.

\section{Acknowledgments}

We acknowledge helpful discussions with H.J. Andrä, L. Hägg, and C.O. Reinhold. This work was supported by the Division of Chemical Sciences, Basic Energy Sciences, Office of Energy Research, U.S. Department of Energy, by the Kansas Center for Advanced Scientific Computing sponsored by the NSF EPSCoR/K*STAR program, and by the National Science Foundation. One of us (JJD) is supported in part by the German Bundesministerium für Bildung, Wissenschaft, Forschung und Technologie under contract no. 13N6776/4. 


\section{REFERENCES}

[1] H.J. Andrä et al., in Proceedings of the XVII International Conference on the Physics of Electronic and Atomic Collisions, Brisbane, Australia, 1991, edited by W.R. MacGillivray, I.E. McCarthy and M.C. Standages (Adam Hilger, N.Y. 1992).

[2] J. Burgdörfer, in Review of Fundamental Processes and Applications of Atoms and Ions, edited by C.D. Lin (World Scientific, Singapore, 1993).

[3] F. Aumayr, in Proceedings of the XIX. International Conference on the Physics of Electronic and Atomic Collisions, Whistler, Canada, 1995, AIP Conference Proceedings 306 (AIP Press, Woodbury, N.Y. 1995).

[4] J. Burgdörfer, P. Lerner, and F. Meyer, Phys. Rev. A 44, 5674 (1991).

[5] J. Burgdörfer, C. Reinhold, and F. Meyer, Nucl. Instrum. Methods Phys. Res. Sect. B 98, 415 (1995).

[6] A. Bárány and C.J. Setterlind, Nucl. Instrum. Methods Phys. Res. Sect. B 98, 184 (1995).

[7] U. Thumm, J. Phys. B 27, 3515 (1994); 28, 91 (1995).

[8] U. Thumm, T. Baştuğ, and B. Fricke, Phys. Rev. A 52, 2955 (1995).

[9] J. Burgdörfer, C. Reinhold, L. Hägg, and F. Meyer, Aust. J. Phys. 49, 527 (1996).

[10] C. Lemell, H.P. Winter, F. Aumayr, J.Burgdörfer, and F. Meyer, Phys. Rev. A 53, 880 (1996).

[11] U. Thumm, Phys. Rev. A 55, 479 (1997).

[12] J. Burgdörfer, E. Kupfer, and H. Gabriel, Phys. Rev. A 35, 4963 (1987).

[13] U. Thumm, J. Phys. B 25, 421 (1992); U. Thumm and J.S. Briggs, Nucl. Instrum. Methods Phys. Res. Sect. B 40/41, 161 (1989); 43, 471 (1989); 47476 (1990). 
[14] A. Borisov, D. Teillet-Billy, and J. Gauyacq, Nucl. Instrum. Methods Phys. Res. Sect. B 78, 49 (1993).

[15] S.A. Deutscher, X.Yang, and Burgdörfer, Phys. Rev. A 55, 466 (1997).

[16] U. Wille, Nucl. Instrum. Methods Phys. Res. Sect. B 100, 303 (1995).

[17] A. Borisov, R. Zimny, D. Teillet-Billy, and J. Gauyacq, Phys. Rev. A 53, 2457 (1996).

[18] P. Kürpick and U. Thumm, Phys. Rev. A 54, 1487 (1996).

[19] P. Nordlander, Phys. Rev. B 53, 4125 (1996).

[20] F. Aumayr, H. Kurz, D. Schneider, M.A. Briere, J.W. McDonald, C.E. Cunningham, and H.P. Winter, Phys. Rev. Lett. 71, 1943 (1993).

[21] H.J. Andrä, A. Simionovici, T. Lamy, A. Brenac, G. A. Pesnelle, Europhys. Lett. 23, 361 (1993).

[22] F.W. Meyer, C.C. Havener, and P.A. Zeijlmans van Emmichoven, Phys. Rev. A 48, 4476 (1993).

[23] J. Limburg, J. Das, S. Schippers, R. Hoekstra, and R. Morgenstern, Phys. Rev. Lett. 73, 786 (1994).

[24] M. Grether, A. Spieler, R. Köhrbrück, and N. Stolterfoht, Phys. Rev. A 52, 426 (1993).

[25] J.-P. Briand, L. de Billy, P. Charles, S. Essabaa, P. Briand, R. Geller, J.-P. Declaux, S. Bliman, and C. Ristori, Phys. Rev. Lett. 65, 159 (1990).

[26] M. Schulz, C. Cocke, S. Hagmann, M. Stöckli, and H. Schmidt-Boecking, Phys. Rev. A 44, 1653 (1991).

[27] H. Winter, J. Phys: Condens. Matter 8, 10149 (1996).

[28] F.W. Meyer, L. Folkerts, H.O. Folkerts, and S. Schippers, Nucl. Instrum. Methods Phys. Res. Sect. B 98, 443 (1995). 
[29] S. Winecki, C. Cocke, D. Fry, and M. Stöckli, Phys. Rev. A 53, 4228 (1996).

[30] Q. Yan, D.M. Zehner, F.W. Meyer, and S. Schippers, Phys. Rev. A 54, 641 (1996).

[31] Ch. Auth, T. Hecht, T. Igel, and H. Winter, Phys. Rev. Lett. 74, 5244 (1995).

[32] J. Limburg, S. Schippers, R. Hoekstra, R. Morgenstern, H. Kurz, F. Aumayr, and HP. Winter, Phys. Rev. Lett. 75, 217 (1995).

[33] Ch. Auth and H. Winter, Phys. Lett. A 217, 119 (1996).

[34] J. Limburg, S. Schippers, R. Hoekstra, R. Morgenstern, H. Kurz, M. Vana, F. Aumayr, HP. Winter, Nucl. Instrum. Methods Phys. Res. Sect. B 115, 237 (1996).

[35] A.G. Borisov, V.Sidis, and H.Winter, Phys. Rev. Lett. 77, 1893 (1996).

[36] L. Hägg, C.O. Reinhold, and J. Burgdörfer, Phys. Rev. A 55, 2097 (1997).

[37] F. Casali and U. Thumm, Bull. Am. Phys. Soc. 41, 1129 (1996).

[38] A. Bárány, G. Astner, H. Cederquist, H. Danared, S. Huldt, P. Hvelplund, A. Johnson, H. Knudsen, L. Liljeby, and K.-G. Rensfeld, Nucl. Instrum. Methods Phys. Res. B 9, 397 (1985).

[39] A. Niehaus, J. Phys. B 19, 2925 (1986).

[40] F.J. García de Abajo and P.M. Echenique, Phys. Rev. B 46, 2663 (1992).

[41] R. Lowndes and D. Martin, Proc. Roy. Soc. London A, 473 (1969).

[42] J. Jackson, Classical Electrodynamics, 2nd ed. (Wiley, N.Y. 1975).

[43] P.J. Jennings, R.O. Jones and W.Weinert, Phys. Rev. B 37, 6113 (1988).

[44] N.F. Mott and M.J. Littleton, Trans. Faraday Soc. 34, 485 (1938).

[45] G.D. Mahan, Phys. Rev. B 21, 4791 (1980).

[46] M. Piacentini and J. Anderegg, Solid State Commun. 38, 191 (1981). 
[47] R. Cowan, The Theory of Atomic Structure and Spectra (University of California Press, Berkeley, 1981).

[48] N.W. Ashcroft and N.D. Mermin, Solid State Physics (W.B. Saunders, Philadelphia 1976).

[49] W.P. O’Brien, Jr. and J.P. Hernandez, Phys. Rev. B 8, 3560 (1974).

[50] G. Molière, Z. Naturforschg.2a 133 (1949).

[51] D. S. Gemmell, Rev. Mod. Phys. 46, 129ff (1974).

[52] H. Winter, Ch. Auth, R. Schuch, and E. Beebe, Phys. Rev. Lett. 71, 1939 (1993).

[53] J. Burgdörfer and F. Meyer, Phys. Rev. A 47, R20 (1993).

[54] H. Kurz, F. Aumayr, HP. Winter, D. Schneider, M.A. Briere, and J.W. McDonald, Phys. Rev. A 49, 4693 (1994). 


\section{FIGURES}

FIG. 1. The band structure of the ionic LiF crystal with a large workfunction of $12 \mathrm{eV}$ and a wide band gap of $14 \mathrm{eV}$. The (polycrystalline) gold target represents a typical metal with a workfunction of about $5 \mathrm{eV}$ and a continuum of unoccupied conduction band states above the Fermi level.

FIG. 2. The linear capture-induced charge distribution $\lambda(x, t)$ trailing the path of the ion (schematically). The capture sequence starts when the incident highly charged ion reaches the critical over-barrier distance at a position $\left(X_{p}, R\right)=\left(x_{0}, R_{c}\right)$ at time $t_{0}$. For our applications to ionic crystals, we assume that electrons are captured from the closest surface anion.

FIG. 3. Classical currents $\vec{j}$ that are driven by the field $\vec{E}=\sigma \vec{j}$ of a capture-induced surface charge restore electric neutrality. A decay time constant $\tau$ can be derived from the macroscopic conductivity $\sigma$ by applying Gauss' theorem to the current $\vec{j}$ and a Gaussian surface given by a hemisphere with the positive excess surface charge in its center.

FIG. 4. The charge state dependent local workfunction $W$ of $\mathrm{LiF}$ is approximated by splitting potentials acting on a bulk atom into an anionic contribution, the ionic binding energy $E_{b i n d}^{q}$, and the Madelung background potential $V_{M a d, b g}$ representing the rest of the bulk. The distance $r_{\text {anion }}^{q}$ denotes the orbital radius of the most loosely bound subshell of the ionic state and is dynamically adjusted to the excess local capture-induced charge $q$.

FIG. 5. Contributions to the total electronic potential $V_{t o t}$ for $50 \mathrm{keV} \mathrm{Xe}{ }^{15+}$ approaching a LiF surface at an angle of $1^{\circ}$ along an axis perpendicular to the surface that includes the projectile nucleus. The projectile is on the incident part of the trajectory at $R=10$ and $q_{p}(R=10)=2.8$.

FIG. 6. Results for $\mathrm{Xe}^{15+}$ at $E_{k i n}=50 \mathrm{keV}$ and an incidence angle of $1^{\circ}$. Time evolution of the projectile charge state for $\mathrm{LiF}$ and $\mathrm{Al}$ surfaces (a). Resonant gain rates on $\mathrm{LiF}$ for the two highest resonantly populated shells (b). Projectile shell occupations on LiF (c). 
FIG. 7. Results for $\mathrm{Xe}^{15+}\left(E_{\text {kin }}=50 \mathrm{keV}\right)$ ions impinging under $1^{\circ}$ grazing incidence conditions on an Al surface. Time evolution of the resonant gain rates for the highest resonantly populated shells (a). Shell occupation (b). Charge exchange primarily takes place via resonant gain into the $n=18$ shell.

FIG. 8. Results for $\mathrm{Xe}^{15+}$ ions impinging with $E_{k i n}=50 \mathrm{keV}$ at an grazing angle of $1^{\circ}$ on $\mathrm{Al}$ and $\mathrm{LiF}$ surfaces. The plot shows the time evolution of the potential barrier $V_{b}$, the target workfunction $W$, and the projectile shell occupations of the most active shells. $V_{b}$ and $W$ display characteristic oscillations on $\mathrm{LiF}$, whereas all potentials evolve smoothly on $\mathrm{Al}$ (see text for details).

FIG. 9. Projectile trajectory in terms of the perpendicular projectile velocity component $v_{z}$ versus $R$ for $20 \mathrm{keV}-\mathrm{Xe}^{15+}$ ions impinging under $1^{\circ}$ on $\mathrm{Al}$ and $\mathrm{LiF}$. The first two curves exhibit standard dynamical COM simulations on these targets. The next two curves show simulation results for the same $\mathrm{Xe}^{15+}$ projectile on $\mathrm{LiF}$ when local surface charges have been disabled in the third and a large "metal" value for the dielectric susceptibility $\epsilon \mapsto \infty$ has been chosen for the fourth curve. $X_{p}=0$ corresponds to the vertex of the full simulation for LiF.

FIG. 10. Experimental [52], simulated staircase COM results [10], and our simulated energy gains (dynamical $\mathrm{COM})$ for $\mathrm{Xe}^{q+}\left(3.7 \mathrm{q} \mathrm{keV}, 1.5^{\circ}\right)$ on an $\mathrm{Al}$ surface. The simple model assumes instantaneous complete neutralization at the first critical distance $R_{c}$ and sets a lower boundary for projectile energy gains. The staircase COM instantaneously transfers one charge unit each time the over-barrier condition is fulfilled. In the dynamical COM continuous charge currents flow between projectile and surface with rates derived from a classical model.

FIG. 11. Experimental [54] and our simulated data (dynamical COM) for very high charge state ions impinging on polycrystalline gold.

FIG. 12. Experimental [28], simulated staircase COM results [10], and our simulated data (dynamical COM) for $150 \mathrm{keV} \mathrm{I}^{q+}$ and $\mathrm{Pb}^{q+}$ ions with charge states $q_{p} \leq 36$ on $\mathrm{Au}$. 
FIG. 13. Experimental energy gains [33] compared with our dynamical COM simulations. Results obtained by neglecting capture-induced local surface charges are labeled as $q_{\text {local }}=0$.

Table I. Static limit $\left(\epsilon_{0}\right)$, optical limit $\left(\epsilon_{\infty}\right)$ and characteristic frequency $\omega_{0}$ used in (2.6) for the dielectric response of $\mathrm{LiF}$ and $\mathrm{KI}$ crystals at $\mathrm{T}=290^{\circ} \mathrm{C} 41$.

\begin{tabular}{|c|c|c|c|}
\hline & $\epsilon_{0}$ & $\epsilon_{\infty}$ & $\omega_{0}$ in $10^{-3}$ a.u. \\
\hline $\mathrm{LiF}$ & 9.00 & 1.93 & 1.39 \\
\hline $\mathrm{KI}$ & 5.09 & 2.65 & 0.46 \\
\hline
\end{tabular}




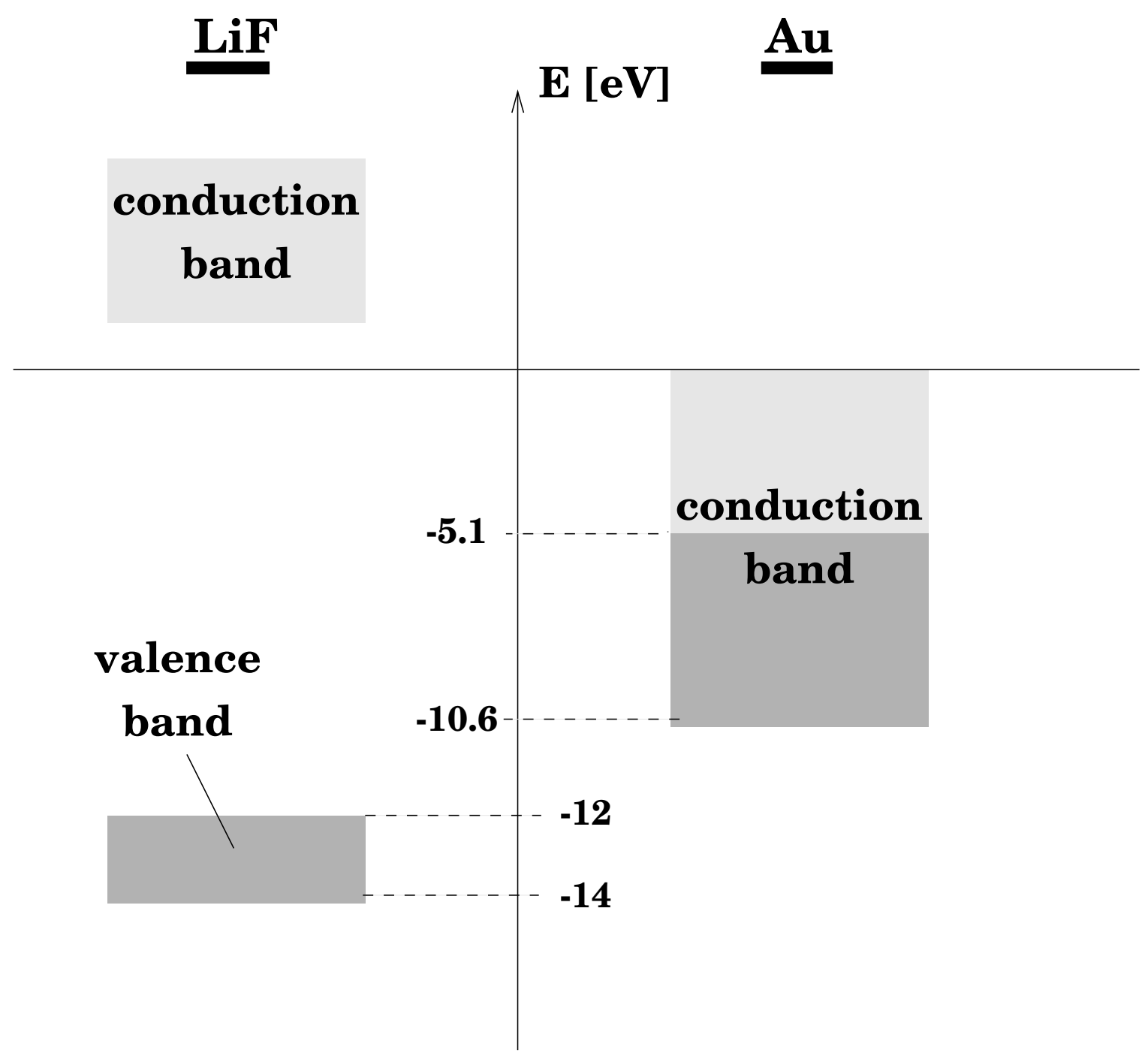

Figure 1 


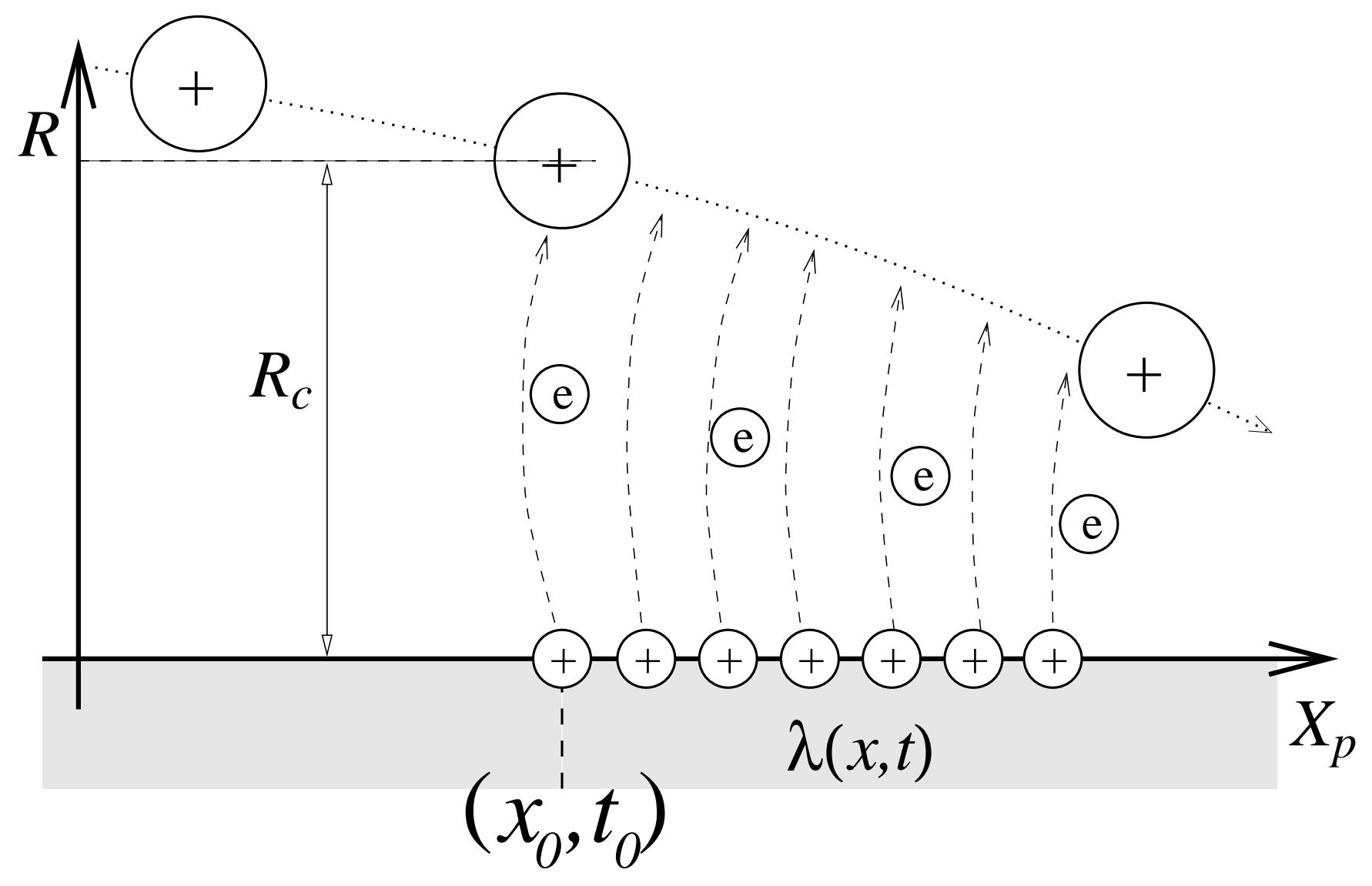

Figure 2 


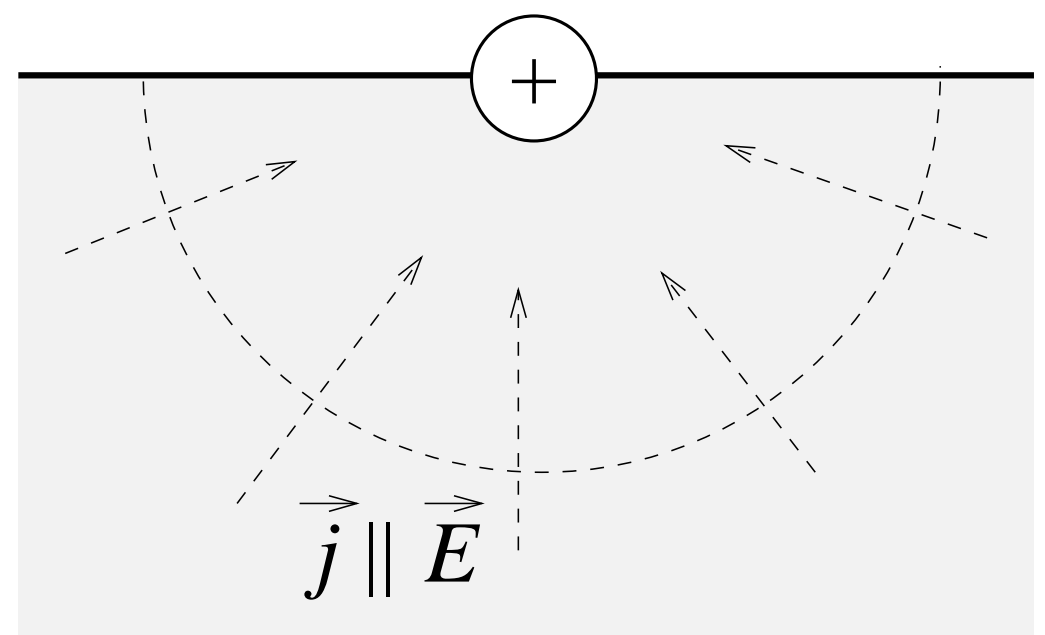

Figure 3 


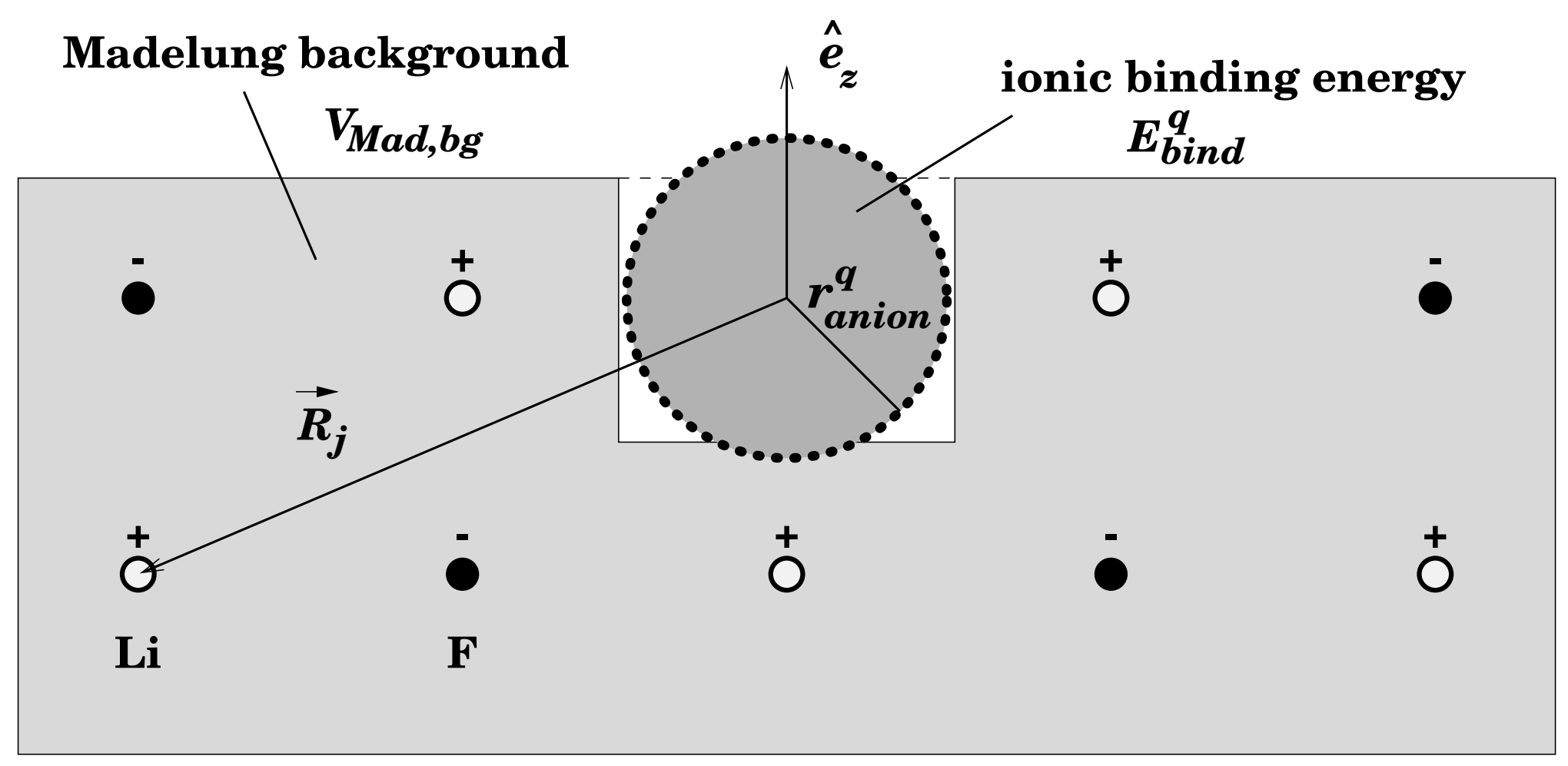

Figure 4 


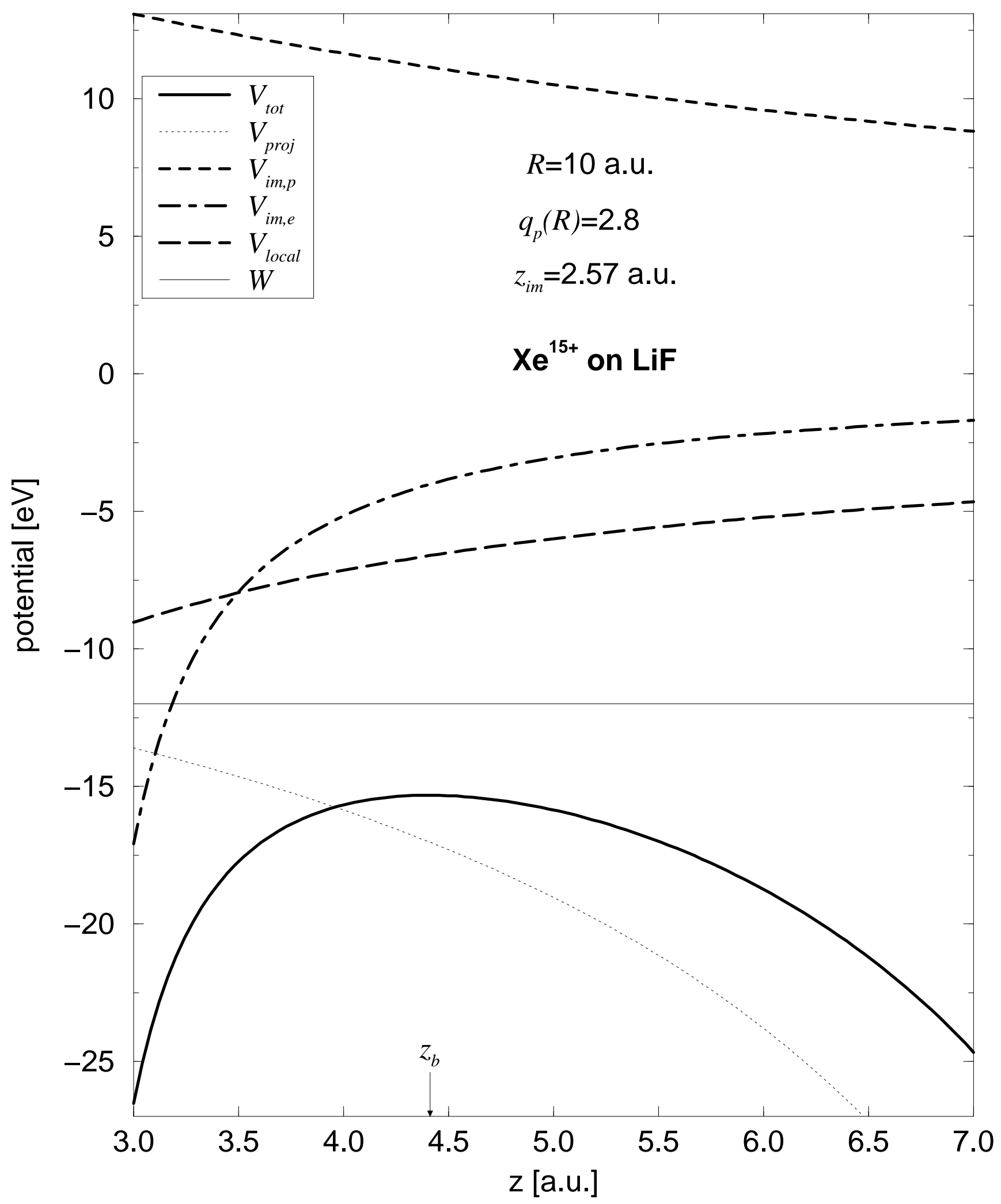

Figure 5 


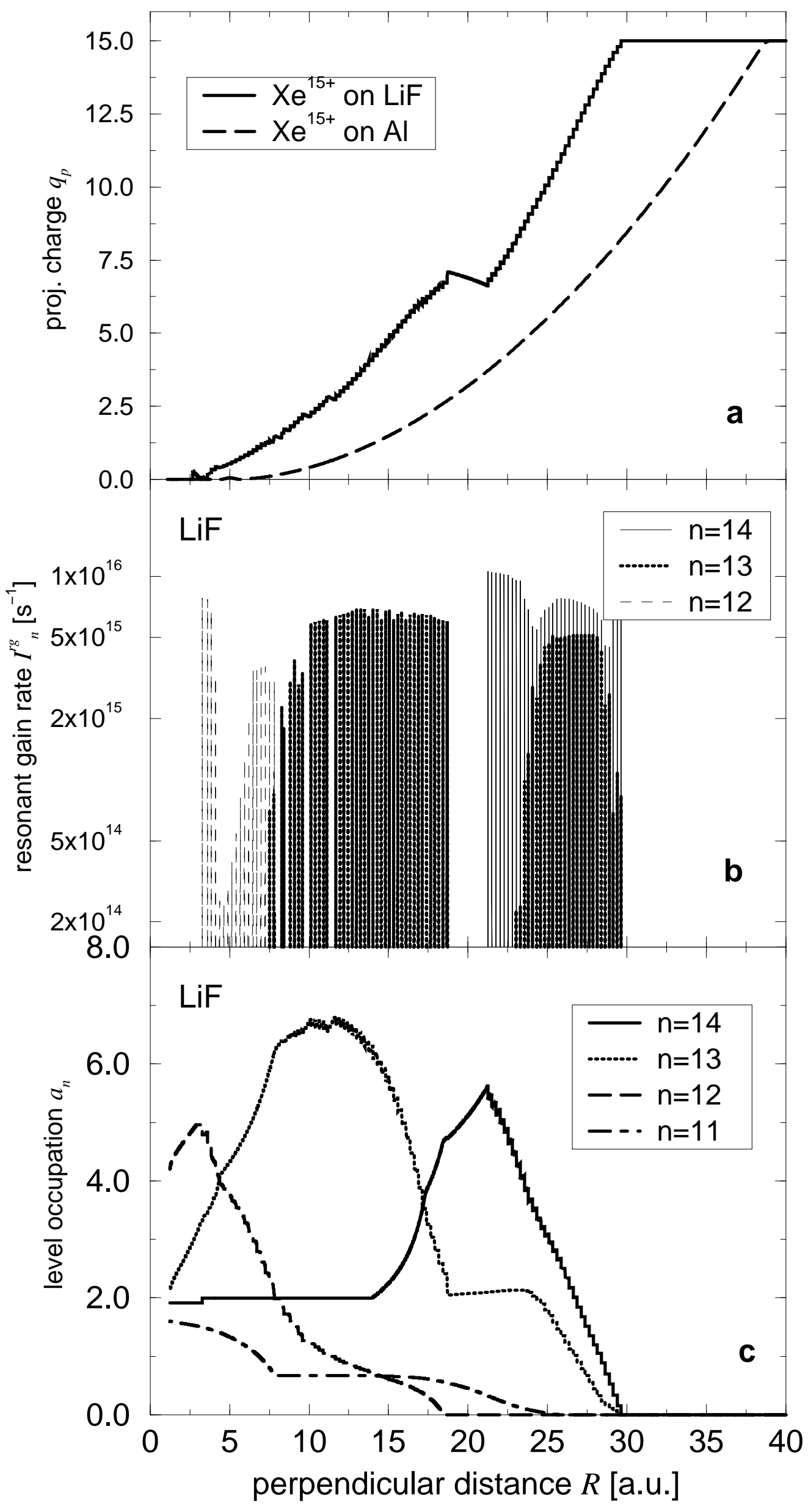

Figure 6 


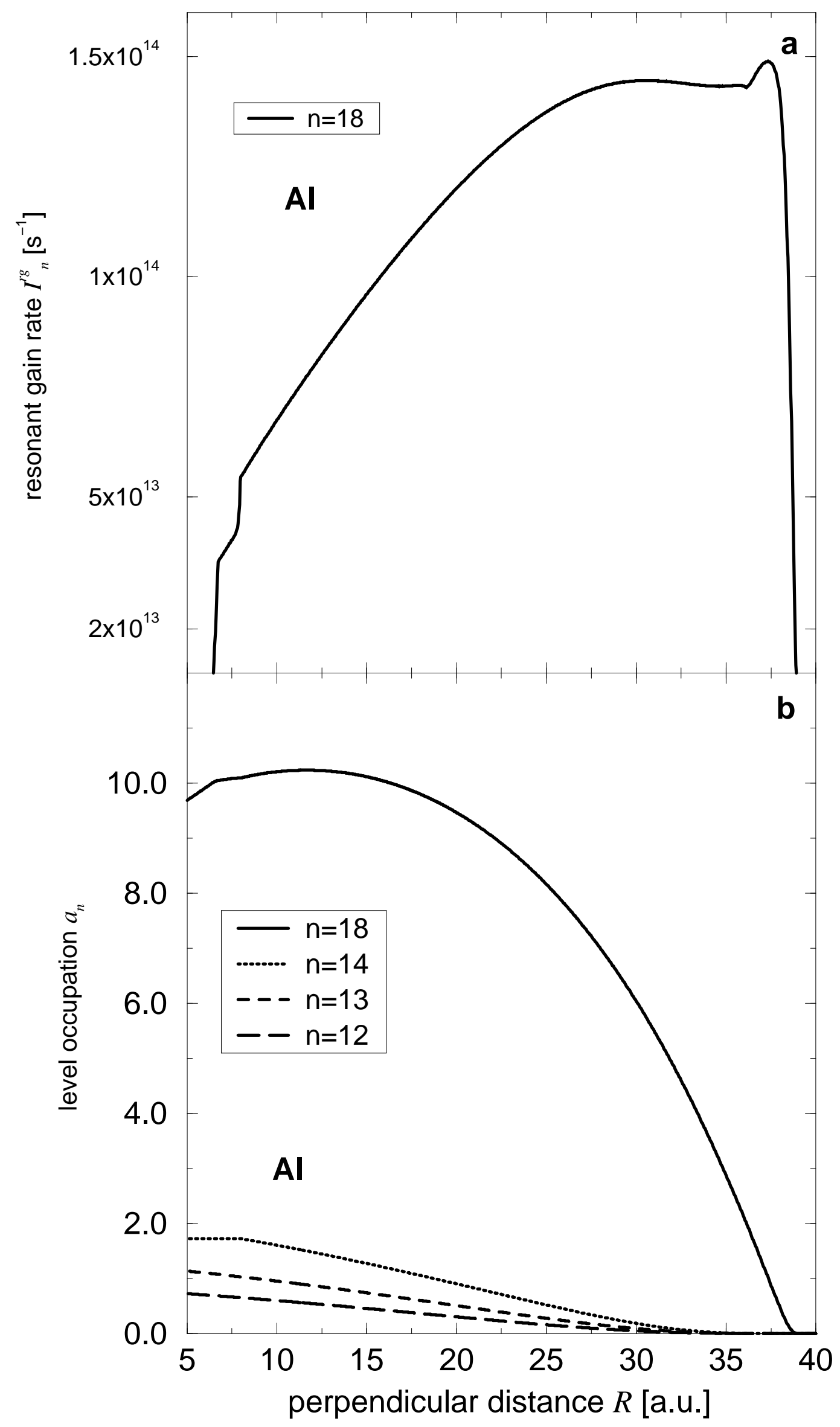

Figure 7 


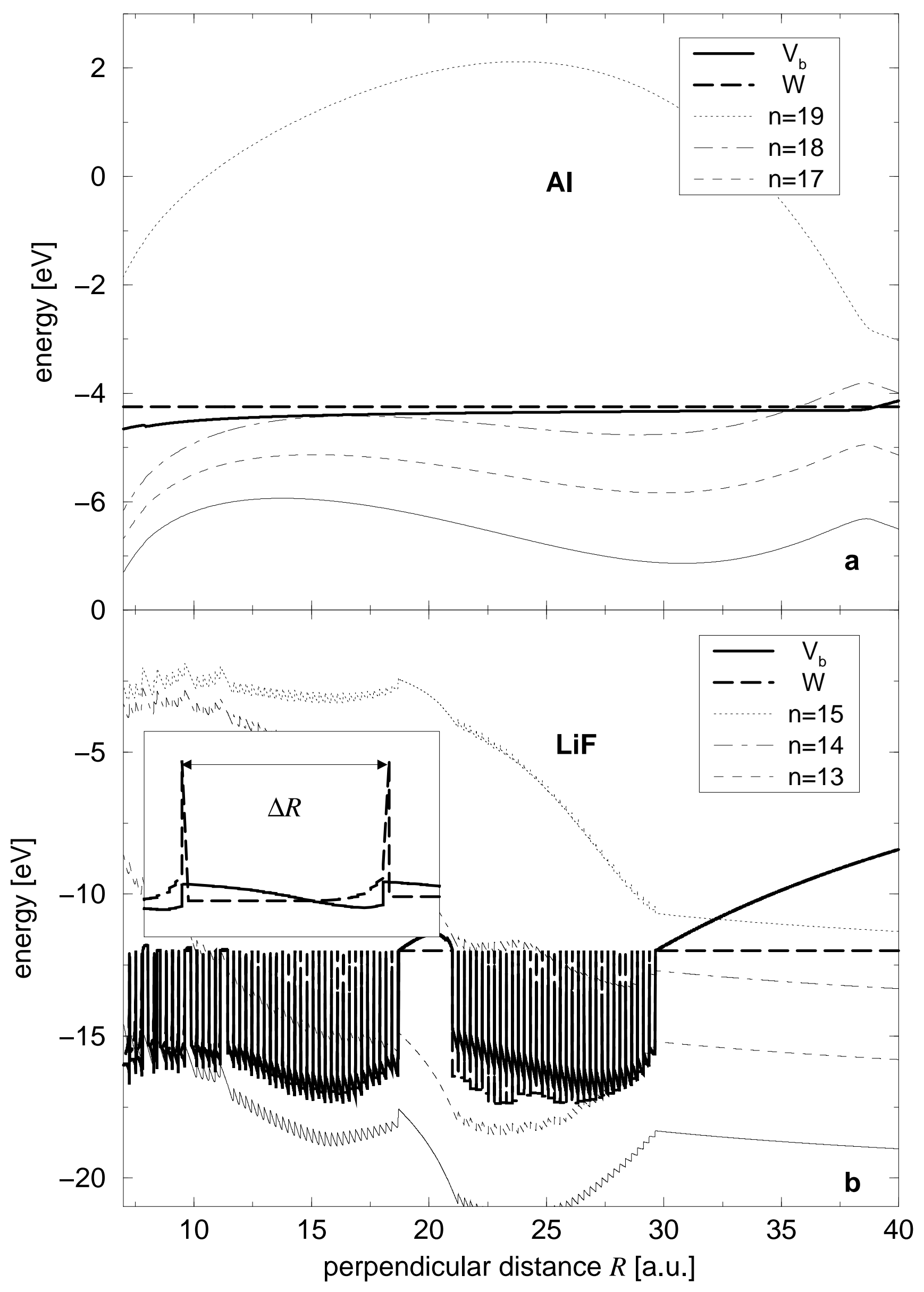

Figure 8 


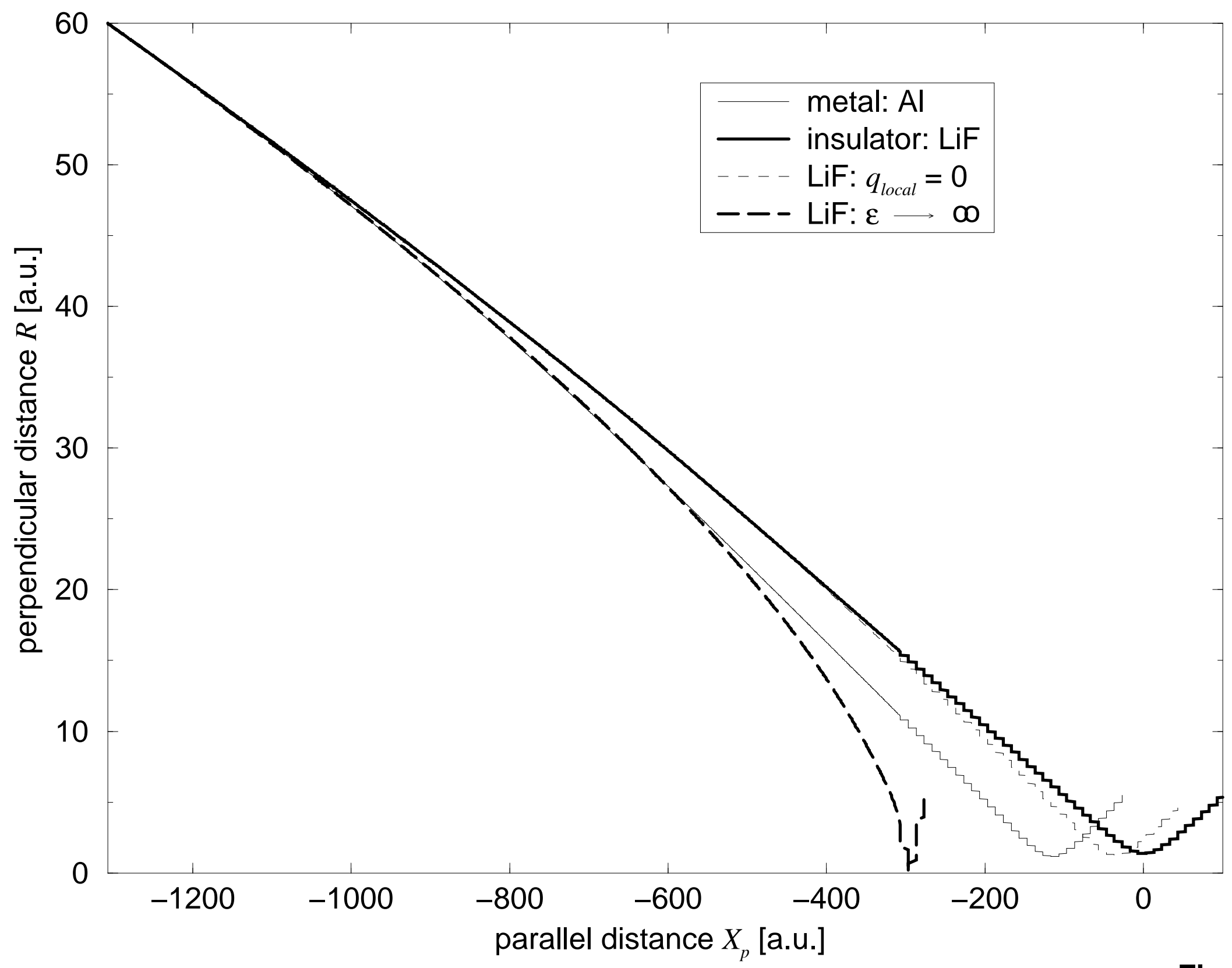

Figure 9 


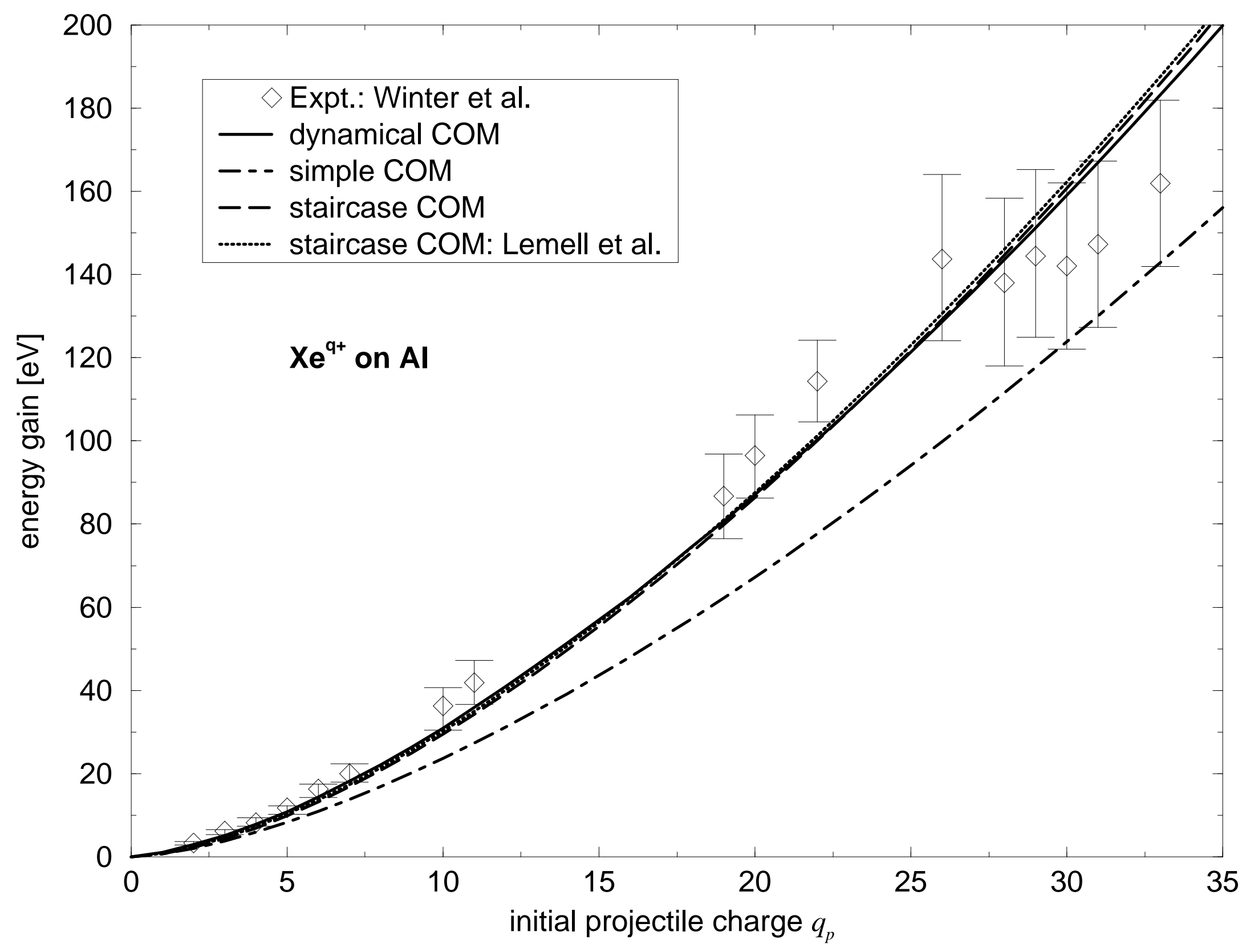

Figure 10 


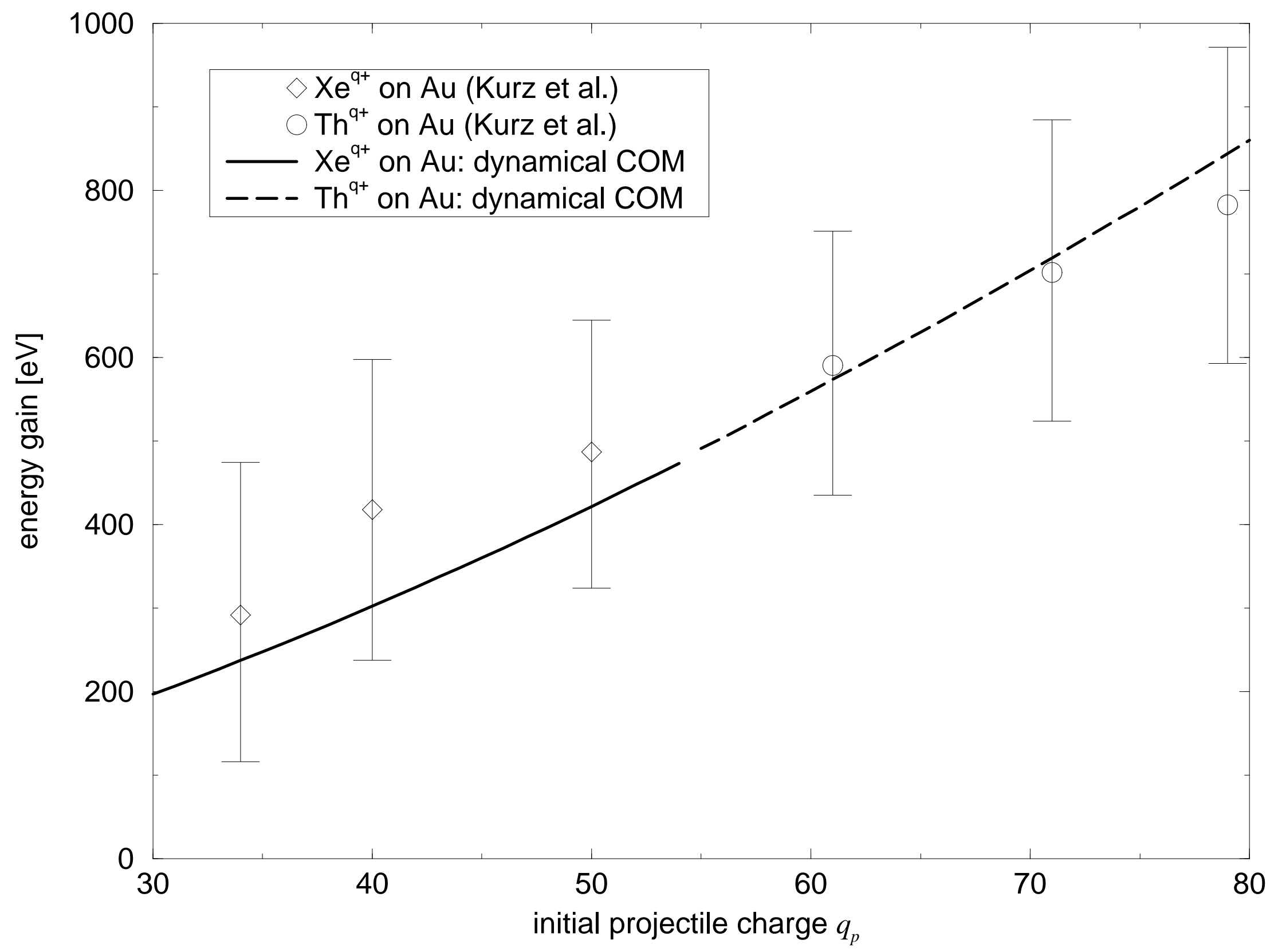

Figure 11 


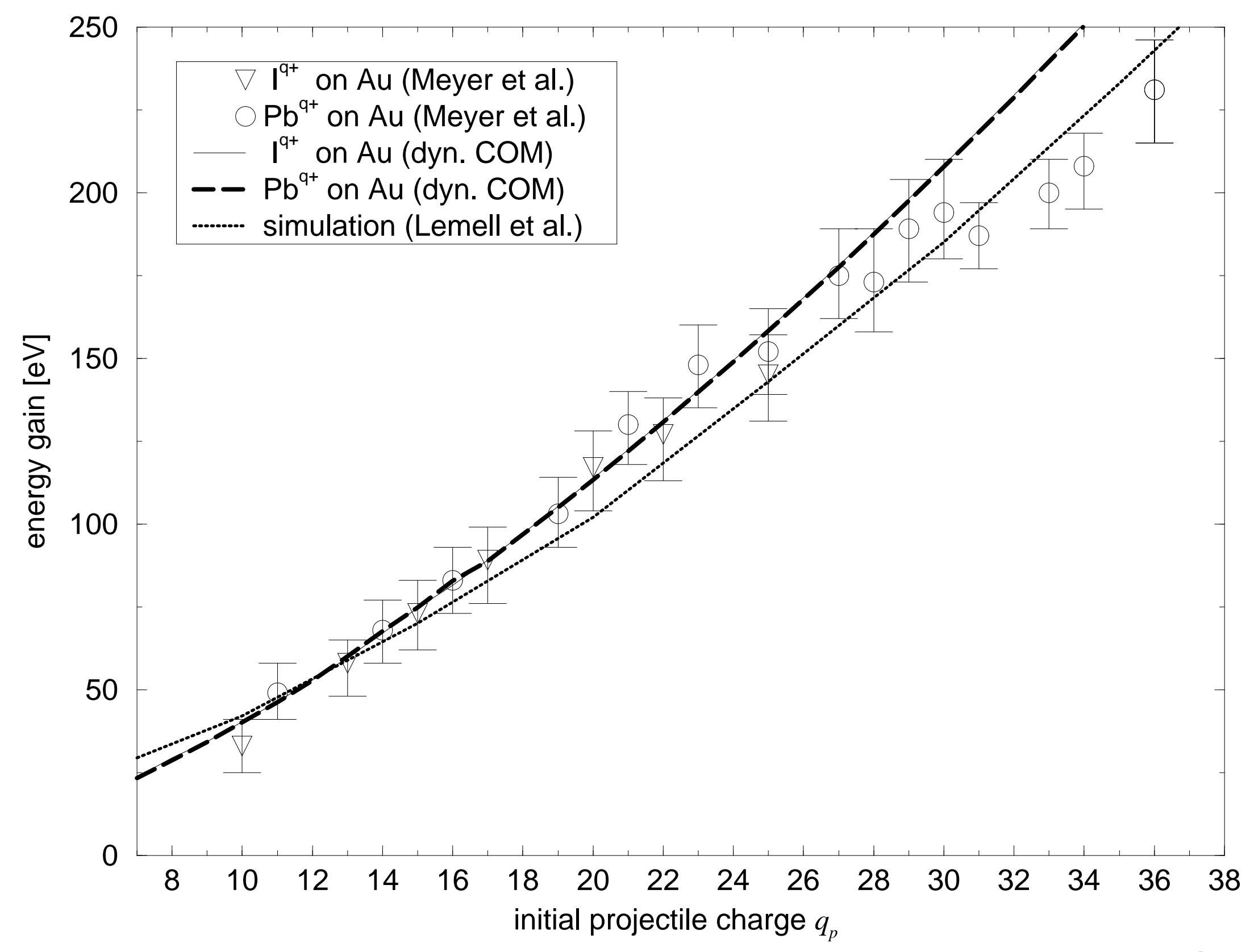

Figure 12 


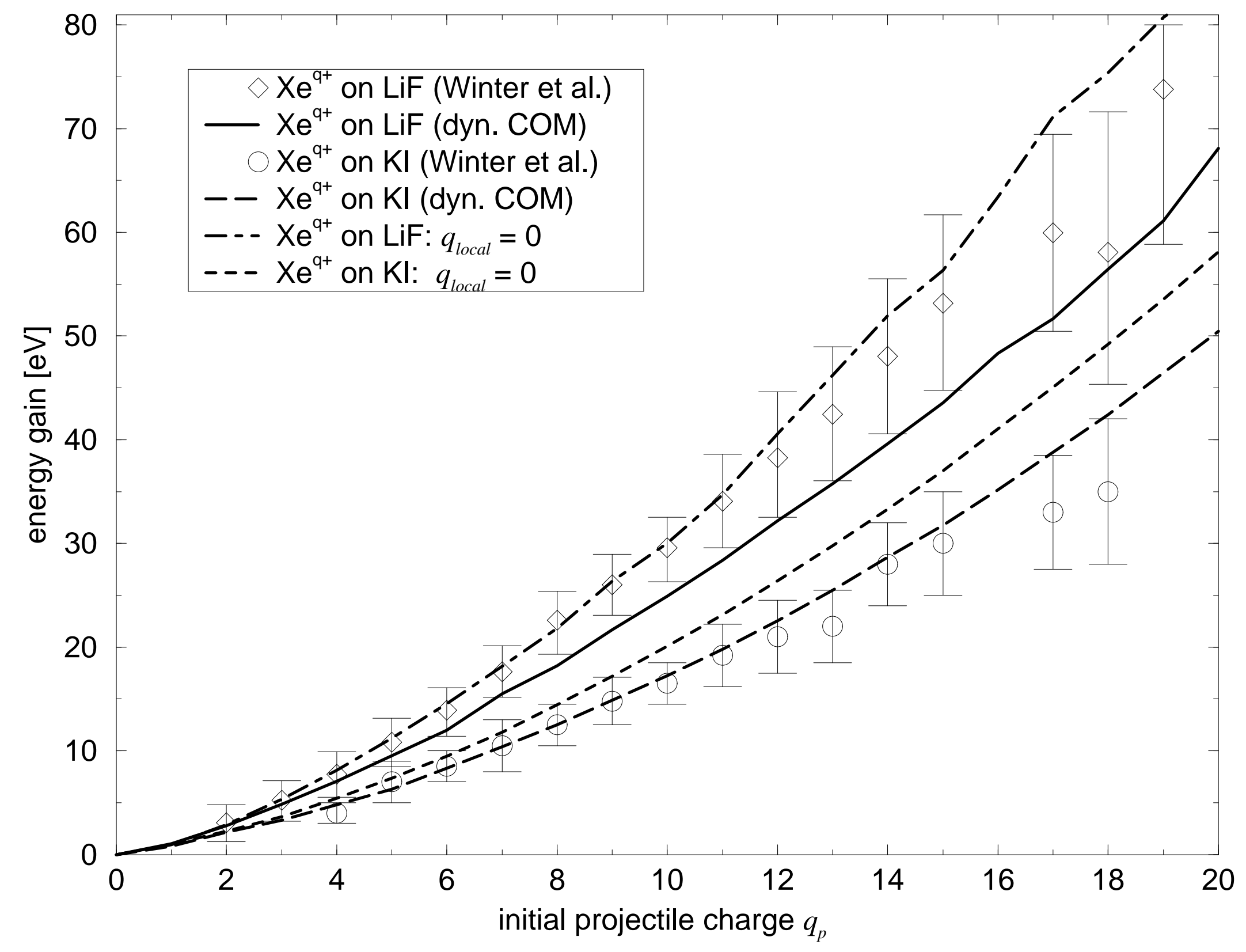

Figure 13 